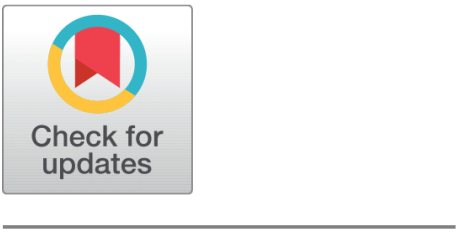

OPEn ACCESS

Received: 09.10.2020

Accepted: 25.01.2021

Published: 01.03 .2021

Citation: Ayub N, Malik A (2021) q-Rung Orthopair Dual Hesitant Fuzzy Bonferron Mean Operators. Indian Journal of Science and Technology 14(6): 582-603. https://d oi.org/10.17485/IJST/v14i6.1838

* Corresponding author.

nausheensuhail7@gmail.com

Funding: None

Competing Interests: None

Copyright: (c) 2021 Ayub \& Malik. This is an open access article distributed under the terms of the Creative Commons Attribution License, which permits unrestricted use, distribution, and reproduction in any medium, provided the original author and source are credited.

Published By Indian Society for Education and Environment (iSee)

ISSN

Print: 0974-6846

Electronic: 0974-5645

\section{q-Rung Orthopair Dual Hesitant Fuzzy Bonferron Mean Operators}

\author{
Nausheen Ayub ${ }^{1 *}$, Aslam Malik ${ }^{1}$ \\ 1 Department of Mathematics, University of the Punjab, Quaid-e-Azam Campus, Lahore, \\ 54590, Pakistan
}

\section{Abstract}

Objectives/Methods: Taking into account the impreciseness and subjectiveness of decision makers (DMs) in complex decision-making situations, the assessment datum over alternatives given by DMs is consistently vague and uncertain. In meantime, to evaluate human's hesitance, the q-rung orthopair dual hesitant fuzzy sets (q-RODHFSs) are defined which are more accurate for manipulation real MADM matters. To merge the datum in q-RODHFSs more precisely, in this research script, some Bonferroni mean (BM) operators in light of q-RODHFSs datum, which includes arbitrary number of being merged arguments, are developed and examined. Findings: Obviously, the novel defined operators can produce much accurate results than already existing methods. Additionally, some important measures of said BM operators are talked about and all the peculiar cases of them are studied which expresses that the BM operator is more dominant than others. Eventually, the MADM algorithm is furnished and the operators are utilized to choose the best alternative under q-rung orthopair dual hesitant fuzzy numbers (q-RODHFNs). Taking advantage of the novel operators and constructed algorithm, the developed operators are utilized in the MADM problems.

Keywords: : Bonferroni mean; Dual BM; q-rung orthopair dual hesitant fuzzy sets; q-rung orthopair dual hesitant fuzzy weighted Bonferroni mean; q-rung orthopair dual hesitant fuzzy weighted dual Bonferroni mean

\section{INTRODUCTION}

Atanassov $^{(1)}$ conferred the concept of intuitionistic fuzzy set (IFS), as an advance form of fuzzy set $(F S)^{(2)}$. Every element enclosed in IFS was interpreted with the degree of membership $\gamma$ and non-membership $\eta$, and their sum is restricted to 1 , in mathematical form can be labeled as $\gamma+\eta \leq 1$. The IFS and hesitant fuzzy sets (HFSs) ${ }^{(3)}$ has appealed many scholars's consideration since its evolution. Likewise, as an impressive MADM technique, Pythagorean fuzzy sets (PFSs) ${ }^{(4)}$ has appeared to outline the uncertainty and fuzziness of the assessment datum. It is also observed that, all the intuitionistic fuzzy decision-making problems are the special case of Pythagorean fuzzy decisionmaking problems, which means that the PFS is more powerful to handle the MADM problems. Wu and Wei ${ }^{(5)}$ developed few Hamacher aggregation operators under PFSs environment to amass PFSs datum. Peng et al. ${ }^{(6)}$ constructed a few novel distance 
measures utilizing PFSs information for use in MADM problems. Wei and Wei ${ }^{(7)}$ introduced a variety of cosine similarity measures for PFSs datum. Yet, practically, there may arise some relationships between more than one arguments, it is clear that previously studied collective operators are not authentic for such purpose. For the solution of such type of problems, the Bonferroni mean $(\mathrm{BM})$ operator $^{(8)}$ as a reputed information collecting tool which have capability to acknowledge the interrelationship of the arguments, have been explored. Liang et al. ${ }^{(9)}$ proposed some BM operators with PFSs information. Most likely, q-ROFS ${ }^{(10)}$ are continuously expansive for the IFS, PFS and these two are its specific cases. Many researchers ${ }^{(11-20)(21)}$ developed a varriety of operators to aggregate the information presented q-ROFSs and its application in MADM. Taking advantage of the classical q-ROFSs, Liu and Liu ${ }^{(22)}$ derived the definition of q-rung orthopair fuzzy linguistic sets (q-ROFLSs) and developed a few power BM aggregation operators for q-ROFLSs datum. Xu et al. ${ }^{(23)}$ illustrated the concept of the q-rung otrhopair dual hesitant fuzzy set (q-RODHFS) and developed a few q-rung dual hesitant fuzzy HM operators for MADM.

Tang et al. (24) developed few Pythagorean fuzzy power aggregation operators and illustrated the idea of dual hesitant Pythagorean fuzzy sets (DHPFSs), as a combination of the PFSs and dual hesitant fuzzy sets (DHFSs) ${ }^{(25,26)(27)}$ also developed some Hamacher aggregation operators utilizing DHPFSs. Jia et al. ${ }^{(28)}$ developed a wide range of distance measures based on connection numbers of set pair analysis with dual hesitant fuzzy sets. Wang et al. ${ }^{(29)}$ developed MM operators under DHPFSs datum. Apparently, there is no exploration led in light of BM operator to fuse q-RODHF information.

In past few years, numerous investigators studied the BM aggregation operators and their applications. The BM operations have the advantage of considering the relationship between the values being fused, thus the fused results are more reasonable and accurate. Clearly, DHq-ROFN is a meaningful tool to express evaluation information. BM operations are good to fuse evaluation information, so it's worth to develop some BM operators under dual hesitant q-rung orthopair fuzzy environments. The main novelty and contribution of our manuscript is developing some new BM operators to aggregate the dual hesitant q-rung orthopair fuzzy information. Evidently, these operators have the following advantages. (1) The DHq-ROFS can not only extend the scope of the assessment information to depict more fuzzy information, but also consider the human's hesitance, thus it is more useful and reasonable to derive decision-making results. (2) The BM operations can consider the relationship between fused arguments, obviously, BM operations are more suitable for handling practical MADM problems. Thus, it is of great significance to propose some new operators based on the dual hesitant q-rung orthopair fuzzy information and BM operations.

In the following text, we have developed a few BM aggregation operators to intertwine the q-RODHF datum. Furthermore, a portion of their alluring properties have additionally been considered and the unique instances of every operator is researched. At last, in light of these effective operators, a decision-making algorithm have been produced and a computative model is delineated to approve the methodology over some similar investigation with the current methodologies. To do as such, the rest of text is composed as pursues. Some basic knowledge about q-ROFSs, q-RODHFSs and BM have been reviewed in Section 2. In Section 3, we have talked about the BM and dual BM operators utilizing q-RODHFSs condition and then developed the q-rung orthopair dual hesitant fuzzy BM(q-RODHFBM) operator, the q-rung orthopair dual hesitant fuzzy weighted BM (q-RODHFWBM) operator, the q-rung orthopair dual hesitant fuzzy dual BM (q-RODHFDBM) operator and the q-rung orthopair dual hesitant fuzzy weighted dual BM (q-RODHFWDBM) operator. In Section 4, we will manufacture the MADM algorithm with q-RODHFNs. In Section 5, we will solve a numerical model for provider choice with q-RODHFNs and gave some similar investigation. Segment 6 , finishes up the discussion with certain comments.

\section{Preliminaries}

\subsection{The q-RUNG ORTHOPAIR FUZZZY SET}

The essential concepts and basic knowledge of q-rung orthopair fuzzy sets (q-ROFSs) ${ }^{(10)}$ are quickly evaluated as pursues.

Definition 2.1. ${ }^{(10)}$ Let $\chi$ be a universal set. A q-ROFS is an item owns the structure

$$
O=\left\{\left\langle x,\left(\gamma_{o}(x), \eta_{o}(x)\right)\right\rangle \mid x \in \chi\right\}
$$

where the mapping $\gamma_{o}(x): \chi \rightarrow[0,1]$ characterizes the membership degree and the mapping $\eta_{o}(x): \chi \rightarrow[0,1]$ characterizes the non-membership degree of the component $x \in \chi$ to $O$, respectively, and, for each $x \in \chi$, it satisfies

$$
\left(\gamma_{o}(x)\right)^{q}+\left(\eta_{o}(x)\right)^{q} \leq 1, \quad q \geq 1
$$

The level of indeterminacy is described as:

$$
\pi_{o}(x)=\sqrt[q]{\left(\gamma_{o}(x)\right)^{q}+\left(\eta_{o}(x)\right)^{q}-\left(\gamma_{o}(x)\right)^{q}\left(\eta_{o}(x)\right)^{q}}
$$


Generally, written as $o=(\gamma, \eta)$ a q-ROFN.

Definition 2.2. ${ }^{(10)}$ Let $o=(\gamma, \eta)$ be a q-ROFN, the score and acuracy function has the form:

$$
\begin{gathered}
L(o)=\frac{1}{2}\left(1+\gamma^{q}-\eta^{q}\right), L(o) \in[0,1] . \\
T(o)=\gamma^{q}+\eta^{q}, \quad T(o) \in[0,1] .
\end{gathered}
$$

to analyze the level of accuracy of the q-ROFN $o=(\gamma, \eta)$ The bigger the value of $T(o)$, the more the level of accuracy of the q-ROFN $o$ is.

Now we describe the comparison rule between two q-ROFNs as pursues:

Definition 2.4. ${ }^{(10)}$ Let $o_{1}=\left(\gamma_{1}, \eta_{1}\right)$ and $o_{2}=\left(\gamma_{2}, \eta_{2}\right)$ be two q-ROFNs, $L\left(o_{1}\right)=\frac{1}{2}\left(1+\gamma_{1}^{q}-\eta_{1}^{q}\right)$ and $L\left(o_{2}\right)=\frac{1}{2}\left(1+\gamma_{2}^{q}-\eta_{2}^{q}\right)$ be score values of $o_{1}$ and $o_{2}$, respectively, and let $T\left(o_{1}\right)=\gamma_{1}^{q}+\eta_{1}^{q}$ and $T\left(o_{2}\right)=\gamma_{2}^{q}+\eta_{2}^{q}$ be the accuracy degrees of $o_{1}$ and $o_{2}$, respectively, then if $L\left(o_{1}\right)<L\left(o_{2}\right)$, then $o_{1} \prec o_{2}$; if $L\left(o_{1}\right)=L\left(o_{2}\right)$, then (1) if $T\left(o_{1}\right)=T\left(o_{2}\right)$, then $o_{1}=o_{2} ;(2)$ if $T\left(o_{1}\right)<T\left(o_{2}\right)$, then $o_{1} \prec o_{2}$.

Definition 2.5. ${ }^{(10)}$ Let $o_{1}=\left(\gamma_{1}, \eta_{1}\right), o_{2}=\left(\gamma_{2}, \eta_{2}\right)$ and $o=(\gamma, \eta)$ be three q-ROFNs, and some basic operations on them are defined as follows:

- $o_{1} \oplus o_{2}=\left(\sqrt[q]{\left(\gamma_{1}\right)^{q}+\left(\gamma_{2}\right)^{q}-\left(\gamma_{1}\right)^{q}\left(\gamma_{2}\right)^{q}}, \eta_{1} \eta_{2}\right)$;

- $o_{1} \otimes o_{2}=\left(\gamma_{1} \gamma_{2}, \sqrt[q]{\left(\eta_{1}\right)^{q}+\left(\eta_{2}\right)^{q}-\left(\eta_{1}\right)^{q}\left(\eta_{2}\right)^{q}}\right)$;

- $\lambda o=\left(\sqrt[q]{1-\left(1-\gamma^{q}\right)^{\lambda}}, \eta^{\lambda}\right), \lambda>0$

- $(o)^{\lambda}=\left(\gamma^{\lambda}, \sqrt[q]{1-\left(1-\eta^{q}\right)^{\lambda}}\right), \lambda>0$

- $o=(\eta, \gamma)$.

\subsection{The q-RUNG ORTHOPAIR DUAL HESITANT FUZZZY SET}

In the light of q-ROFSs ${ }^{(10)}$ and dual hesitant fuzzy sets ${ }^{(25)},{ }^{(26)} \mathrm{Xu}$ et al. ${ }^{(23)}$ introduced the idea and primary operations of the q-rung orthopair dual hesitant fuzzy sets (q-RODHFSs).

Definition 2.6. ${ }^{(23)}$ For any universal set $\chi$, a q-rung orthopair dual hesitant fuzzy set (q-RODHFS) on $\chi$ is given as:

$$
D=\left(\left\langle x, h_{O}(x), g_{O}(x)\right\rangle \mid x \in \chi\right)
$$

Where $h_{O}(x)=\cup_{\rho \in h_{O}}\{\rho\}$ and $g_{O}(x)=\cup_{\kappa \in g_{O}}\{\kappa\}$ are two objects, also $0 \leq h_{O}(x), g_{O}(x) \leq 1$, telling the membership (favorable) degrees and non-membership (unfavorable) degrees of the element $x \in \chi$ to the set $D$ respectively, with the criteria:

$$
\cup_{\rho \in h}(\max (\rho))^{q}+\cup_{\kappa \in g}(\max (\kappa))^{q} \leq 1
$$

Where $\rho \in h_{o}(x), \kappa \in g_{o}(x)$ for all $x \in \chi$. Instantly, the pair $d(x)=\left(h_{o}(x), g_{o}(x)\right)$ is called a q-rung orthopair dual hesitant fuzzy number (q-RODHFN) simply written as $d=(h, g)$, with the criteria: $\rho \in h, \kappa \in g, 0 \leq \rho, \kappa \leq 1$ and $\cup_{\rho \in h}(\max (\rho))^{q}+$ $\cup_{\kappa \in g}(\max (\kappa))^{q} \leq 1$.

Moreover, the relationship among q-RODHFNs could be communicated as:

Definition 2.7. ${ }^{(23)}$ For a q-RODHFN $d=\left(h_{o}, g_{o}\right)$, the score and accuracy functions are given as $S(d)=\frac{1}{2}\left(1+\frac{1}{\# h} \sum_{\rho \in h} \rho^{q}-\right.$ $\left.\frac{1}{\# g} \sum_{\kappa \in g} \kappa^{q}\right)$ and $T(d)=\left(\frac{1}{\sharp h} \sum_{\rho \in h} \rho^{q}+\frac{1}{\sharp g} \sum_{\kappa \in g} \kappa^{q}\right)$, where $\sharp h$ and $\sharp g$ are the numbers of the elements in $h$ and $g$ respectively, then, Let $d_{i}=\left(h_{i}, g_{i}\right)(i=1,2)$ be any two q-RODHFNs, we have these comparison rules: if $S\left(d_{1}\right)>S\left(d_{2}\right)$, then $d_{1} \succ d_{2}$; if $S\left(d_{1}\right)=S\left(d_{2}\right)$, then: (1) if $T\left(d_{1}\right)=T\left(d_{2}\right)$, then $d_{1}=d_{2} ;(2)$ if $T\left(d_{1}\right)>T\left(d_{2}\right)$, then $d_{1} \succ d_{2}$.

Definition 2.8. ${ }^{(23)}$ Let $d_{1}=\left(h_{1}, g_{1}\right), d_{2}=\left(h_{2}, g_{2}\right)$ and $d=(h, g)$ be three q-RODHFNs, then, the basic working rules on the q-RODHFNs are defined as:

$$
\begin{aligned}
& d_{1} \oplus d_{2}=U_{\rho_{1} \in h_{1}, \kappa_{1} \in g_{1}, \rho_{2} \in h_{2}, \kappa_{2} \in g_{2}}\left\{\left\{\sqrt[q]{\left(\rho_{1}\right)^{q}+\left(\rho_{2}\right)^{q}-\left(\rho_{1}\right)^{q}\left(\rho_{2}\right)^{q}}\right\},\left\{\kappa_{1} \kappa_{2}\right\}\right. \\
& d_{1} \otimes d_{2}=\mathrm{U}_{\rho_{1} \in h_{1}, \kappa_{1} \in g_{1}, \rho_{2} \in h_{2}, \kappa_{2} \in g_{2}}\left\{\left\{\rho_{1} \rho_{2}\right\},\left\{\sqrt[q]{\left(\kappa_{1}\right)^{q}+\left(\kappa_{2}\right)^{q}-\left(\kappa_{1}\right)^{q}\left(\kappa_{2}\right)^{q}}\right\}\right\}
\end{aligned}
$$




$$
\begin{aligned}
& \lambda d=U_{\rho \in h, K \in g}\left\{\left\{\sqrt[q]{1-\left(1-\rho^{q}\right)^{\lambda}}\right\},\left\{\kappa^{\lambda}\right\}\right\}, \lambda>0 \\
& d^{\lambda}=U_{\rho \in h, K \in g}\left\{\left\{\rho^{\lambda}\right\},\left\{\sqrt[q]{1-\left(1-\kappa^{q}\right)^{\lambda}}\right\}\right\}, \lambda>0
\end{aligned}
$$

\subsection{BM OPERATORS}

Bonferroni $^{(8)}$ proposed the Bonferroni mean (BM) operator.

Definition 2.9. ${ }^{(8)}$ Suppose $s, t \geq 0$, and $b_{i}(i=1,2, \ldots, \tau)$ be nonnegative real numbers. If

$$
B M^{s, t}\left(b_{1}, b_{2}, \ldots, b_{\tau}\right)=\left(\frac{1}{\tau(\tau-1)} \sum_{\substack{i, j=1 \\ i \neq j}}^{\tau} b_{i}^{s} b_{j}^{t}\right)^{\frac{1}{s+t}}
$$

Then we called $B M^{s, t}$ the Bonferroni mean (BM) operator.

\subsection{The q-RODHFBM OPERATOR}

This segment stretches out BM and to fuse the q-RODHFNs, we will introduce the q-rung orthopair dual hesitant fuzzy Bonferroni mean (q-RODHFBM) operator, besides, some valuable properties of q-RODHFBM operator are talked about.

Definition 2.10. Let $d_{j}=\left(h_{j}, g_{j}\right)(j=1,2, \ldots, \tau)$ be an assortment of q-RODHFNs. The q-rung orthopair dual hesitant fuzzy Bonferroni mean (q-RODHFBM) can be composed as:

$$
q-\operatorname{RODHFBM} M^{s, t}\left(d_{1}, d_{2}, \ldots, d_{\tau}\right)=\left(\frac{1}{\tau(\tau-1)}\left(\oplus_{i \neq j}^{\tau}\left(d_{i}^{s} \otimes d_{j}^{t}\right)\right)\right)^{\frac{1}{s+t}}
$$

Theorem 1. Let $d_{j}=\left(h_{j}, g_{j}\right)(j=1,2, \ldots, \tau)$ be a list of q-RODHFNs. We can intertwine all the q-RODHFNs datum by utilizing the q-RODHFBM operator, the intertwined outcomes can be communicated in Eq.8, as pursues.

$$
\begin{aligned}
& q-\operatorname{RODHFBM} M^{s, t}\left(d_{1}, d_{2}, \ldots, d_{\tau}\right)=\left(\frac{1}{\tau(\tau-1)}\left(\oplus_{\substack{i, j=1 \\
i \neq j}}^{\tau}\left(d_{i}^{s} \otimes d_{j}^{t}\right)\right)\right)^{\frac{1}{s+t}} \\
& =U_{\rho_{i} \in h_{i}, \rho_{j} \in h_{j}, \kappa_{i} \in g_{i}, \kappa_{j} \in g_{j}}\left\{C^{q} \sqrt{\left.1-\prod_{i, j=1}^{\tau}\left(1-\left(\rho_{i}^{S} \rho_{j}^{t}\right)^{q}\right)^{\frac{1}{\tau(\tau-1)}}\right)^{\frac{1}{s+t}}}\right. \\
& \sqrt[q]{1-\left(1-\left(\prod_{\substack{\tau, j=1 \\
i \neq j}}^{\tau}\left(1-\left(1-\kappa_{i}^{q}\right)^{s}\left(1-\kappa_{j}^{q}\right)^{t}\right)\right)^{\frac{1}{\tau(\tau-1)}}\right)^{\frac{1}{s+t}}}
\end{aligned}
$$

Proof. The proof is simple and obvious from definition (2.8). 
Example 2.1. Let $d_{1}=\{\{0.3,0.4\},\{0.5\}\}, d_{2}=\{\{0.7\},\{0.1,0.2,0.6\}\}$, and $d_{3}=\{\{0.6\},\{0.3\}\}$ be three q-RODHFNs, and let $s=1, t=1$ and $q=3$ then according to Eq.8, we have

$$
\begin{aligned}
& q-\operatorname{RODHFBM} M^{s, t}\left(d_{1}, d_{2}, \ldots, d_{\tau}\right)=\left(\frac{1}{\tau(\tau-1)}\left(\oplus_{\substack{i, j=1 \\
i \neq j}}^{\tau}\left(d_{i}^{s} \otimes d_{j}^{t}\right)\right)\right)^{\frac{1}{s+t}} \\
& U_{\rho_{i} \in h_{i}, \rho_{j} \in h_{j}, k_{i} \in g_{i}, \kappa_{j} \in g_{j}}\left\{\left(q \sqrt{\left.1-\prod_{i, j=1}^{\tau}\left(1-\left(1-\rho_{i}^{s} \rho_{j}^{t}\right)^{q}\right)^{\frac{1}{\tau(\tau-1)}}\right)^{\frac{1}{s+t}}}\right.\right.
\end{aligned}
$$

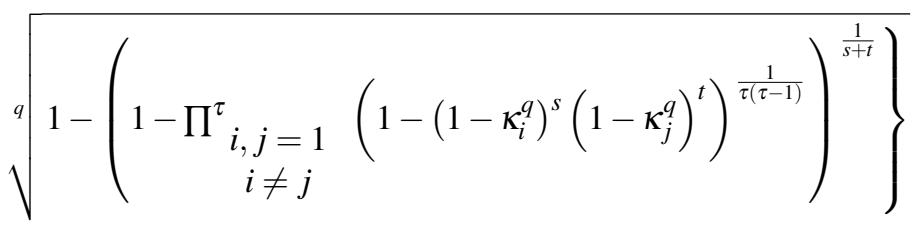

$$
\begin{aligned}
& \rho_{1}=q-\operatorname{RODHFBM} M^{1,1}(0.3,0.7,0.6)=\left(\frac{1}{3(3-1)}\left(\otimes_{i, j=1}^{3}\left(d_{i}^{1} \otimes d_{j}^{1}\right)\right)\right)^{\frac{1}{1+1}} \\
& =\left(\sqrt[3]{1-\prod_{i, j=1}^{3}\left(1-\left(1-\rho_{i} \rho_{j}\right)^{3}\right)^{\frac{1}{3(3-1)}}}\right)^{\frac{1}{2}}=0.5585 \\
& \kappa_{1}=q-\operatorname{RODHFBM} M^{1,1}(0.5,0.1,0.3) \\
& =\sqrt[3]{1-\left(1-\prod_{i, j=1}^{3}\left(1-\left(1-\kappa_{i}^{3}\right)^{1}\left(1-\kappa_{j}^{3}\right)^{1}\right)^{\frac{1}{3(3-1)}}\right)^{\frac{1}{2}}}=0.3559
\end{aligned}
$$

The fused outcomes of the membership function $\rho$, are displayed as below.

Similarly, we can find $\rho_{2}=\mathrm{q}-\operatorname{RODHFBM}(0.4,0.7,0.6)=0.8013$, and $\rho=\{0.5585,0.8013\}$. For the unfavorable (nonmembership) function $\kappa$, the fused outcomes are displayed as. Alike, the values of $\kappa_{2}$, and $\kappa_{3}$, are $\kappa_{2}=\mathrm{q}-\mathrm{RODHFBM}(0.5$, $0.2,0.3)=0.3557, \kappa_{3}=q-\operatorname{RODHFBM}(0.5,0.6,0.3)=0.4911$, so we can find $\kappa=\{0.3559,0.3557,0.4911\}$. Therefore,

$$
q-\operatorname{RODHFBM}\left(d_{1}, d_{2}, d_{3}\right)=\{\{0.5585,0.8013\},\{0.3559,0.3557,0.4911\}\}
$$

By adjusting the estimations of parameter $s, t$ and $q$, some unique instances of q-RODHFBM operator are discussed as pursues.

(1) For parameter q, there arise the accompanying exceptional cases

Remark 1. When $q=1$, the q-RODHFBM operator will turn to dual hesitant fuzzy BM (DHFBM) operator given as:

$$
\begin{aligned}
& \operatorname{DHFBM}^{s, t}\left(d_{1}, d_{2}, \ldots, d_{\tau}\right)=\left(\frac{1}{\tau(\tau-1)}\left(\oplus_{\substack{i, j=1 \\
i \neq j}}^{\tau}\left(d_{i}^{s} \otimes d_{j}^{t}\right)\right)\right)^{\frac{1}{s+t}} \\
& =\cup_{\rho_{i} \in h_{i}, \kappa_{i} \in g_{i}, \rho_{j} \in h_{j}, \kappa_{j} \in g_{j}}\left\{\left(1-\prod_{\substack{i, j=1 \\
i \neq j}}^{\tau}\left(1-\rho_{i}^{s} \rho_{j}^{t}\right) \frac{1}{\tau(\tau-1)}\right) \frac{1}{s+t}\right. \\
& \left.1-\left(1-\prod_{\substack{i, j=1 \\
i \neq j}}^{\tau}\left(1-\left(1-\kappa_{i}\right)^{s}\left(1-\kappa_{j}\right)^{t}\right)^{\frac{1}{\tau(\tau-1)}}\right) \frac{1}{s+t}\right\}
\end{aligned}
$$


Remark 2. When $q=2$, the q-RODHFBM operator will turn into dual hesitant Pythagorean fuzzy BM (DHPFBM) operator given as:

$$
\begin{aligned}
& \operatorname{DHFBM}^{s, t}\left(d_{1}, d_{2}, \ldots, d_{\tau}\right)=\left(\frac{1}{\tau(\tau-1)}\left(\oplus_{\substack{i, j=1 \\
i \neq j}}^{\tau}\left(d_{i}^{s} \otimes d_{j}^{t}\right)\right)\right)^{\frac{1}{s+t}} \\
& =\cup_{\rho_{i} \in h_{i}, \kappa_{i} \in g_{i}, \rho_{j} \in h_{j}, \kappa_{j} \in g_{j}}\left\{\left(\sqrt{1-\prod_{\substack{i, j=1 \\
i \neq j}}^{\tau}\left(1-\left(\rho_{i}^{s} \rho_{j}^{t}\right)^{2}\right)^{\frac{1}{\tau(\tau-1)}}}\right)^{\frac{1}{s+t}},\right. \\
& \sqrt{1-\left(1-\prod_{\substack{i, j=1 \\
i \neq j}}^{\tau}\left(1-\left(1-\kappa_{i}^{2}\right)^{s}\left(1-\kappa_{j}^{2}\right)^{t}\right)^{\frac{1}{\tau(\tau-1)}} \frac{1}{s+t}\right\}}
\end{aligned}
$$

(2) For parameter $s$ and $t$, now we discuss these important cases.

Remark 3. When $t \rightarrow 0$, then the q-RODHFBM will turn into the q-rung orthopair dual hesitant fuzzy arithmetic mean (q-RODHFAM) as shown below:

$$
\begin{aligned}
& q-\operatorname{RODHFAM} M^{s, 0}\left(d_{1}, d_{2}, \ldots, d_{\tau}\right)=\left(\frac{1}{\tau(\tau-1)}\left(\oplus_{\substack{i, j=1 \\
i \neq j}}^{\tau}\left(d_{i}^{s} \otimes d_{j}^{0}\right)\right)\right) \frac{1}{s+t} \\
& =\cup_{\rho_{i} \in h_{i}, \kappa_{i} \in g_{i}}\left\{\left(\sqrt[q]{1-\prod_{i=1}^{\tau}\left(1-\left(\rho_{i}^{s}\right)^{q}\right) \frac{1}{\tau}}\right) \frac{1}{s}, \sqrt[q]{1-\left(1-\prod_{i=1}^{\tau}\left(1-\left(1-\kappa_{i}^{q}\right)^{s}\right) \frac{1}{\tau}\right.} \frac{1}{s}\right\}
\end{aligned}
$$

Remark 4. If $s=2$ and $t \rightarrow 0$, then the q-RODHFBM will turn to the q-rung orthopair dual hesitant fuzzy square mean (qRODHFSM) as shown below:

$$
\begin{aligned}
& q-\operatorname{RODHFAM} M^{2,0}\left(d_{1}, d_{2}, \ldots, d_{\tau}\right)=\left(\frac{1}{\tau(\tau-1)}\left(\oplus_{\substack{i, j=1 \\
i \neq j}}^{\tau}\left(d_{i}^{2} \otimes d_{j}^{0}\right)\right)\right)^{\frac{1}{2+0}} \\
& \left.=\cup_{\rho_{i} \in h_{i}, \kappa_{i} \in g_{i}}\left\{\left(\sqrt[q]{1-\prod_{\substack{i, j=1 \\
i \neq j}}^{\tau}\left(1-\left(\rho_{i}^{2}\right)^{q}\right) \frac{1}{\tau}}\right)^{\frac{1}{2}}, \sqrt[q]{1-\left(1-\prod_{\substack{i, j=1 \\
i \neq j}}^{\tau}\left(1-\left(1-\kappa_{i}^{q}\right)^{2}\right)^{\frac{1}{\tau}}\right.}\right)^{\frac{1}{2}}\right\}
\end{aligned}
$$

Remark 5. If $s=1$ and $t \rightarrow 0$, then the q-RODHFBM will turn into the q-rung orthopair dual hesitant fuzzy geometric mean (q-RODHFGM) operator as shown below:

$$
\begin{aligned}
& q-\operatorname{RODHFAM} M^{1,0}\left(d_{1}, d_{2}, \ldots, d_{\tau}\right)=\left(\frac{1}{\tau(\tau-1)}\left(\oplus_{\substack{i, j=1 \\
i \neq j}}^{\tau}\left(d_{i}^{1} \otimes d_{j}^{0}\right)\right)\right)^{\frac{1}{1}} \\
& =\cup_{\rho_{i} \in h_{i}, \kappa_{i} \in g_{i}}\left\{\sqrt[q]{1-\prod_{i=1}^{\tau}\left(1-\left(\rho_{i}\right)^{q}\right)} \frac{\sqrt[1]{\tau}}{\sqrt[q]{\left(\prod_{i=1}^{\tau}\left(\kappa_{i}\right)^{q}\right)^{\frac{1}{\tau}}}}\right\}
\end{aligned}
$$


Remark 6. When $s=t=1$, then the q-RODHFBM will turn into the q-rung orthopair dual hesitant fuzzy interrelated square mean (q-RODHFISM) operator as shown below

$$
\begin{aligned}
& q-\operatorname{RODHFAM} M^{1,1}\left(d_{1}, d_{2}, \ldots, d_{\tau}\right)=\left(\frac{1}{\tau(\tau-1)}\left(\oplus_{\substack{i, j=1 \\
i \neq j}}^{\tau}\left(d_{i}^{1} \otimes d_{j}^{1}\right)\right)\right)^{\frac{1}{2}} \\
& =\cup_{\rho_{i} \in h_{i}, \kappa_{i} \in g_{i}, \rho_{j} \in h_{j}, \kappa_{j} \in g_{j}}\left\{\left(\sqrt[q]{1-\prod_{\substack{i, j=1 \\
i \neq j}}^{\tau}\left(1-\left(\rho_{i} \rho_{j}\right)^{q}\right)} \frac{1}{\tau(\tau-1)}\right) \frac{1}{2},\right. \\
& \left.\sqrt[q]{1-\left(1-\prod_{\substack{i, j=1 \\
i \neq j}}^{\tau}\left(1-\left(1-\kappa_{i}^{q}\right)\left(1-\kappa_{j}^{q}\right)\right) \frac{1}{\tau(\tau-1)}\right)} \frac{1}{2}\right\}
\end{aligned}
$$

\subsection{THE q-RODHFWBM OPERATOR}

To get better results in MADM, it's good to take weighted attributes. In this segment we will introduce the q-rung orthopair dual hesitant fuzzy weighted Bonferroni mean (q-RODHFWBM) operator by this way.

Definition 2.11. Let $d_{j}=\left(h_{j}, g_{j}\right)(j=1,2, \ldots, \tau)$ be an assortment of q-RODHFNs with the weight vector $w=$ $\left(w_{1}, w_{2}, \ldots, w_{\tau}\right)^{T}$, there by satisfying $w_{i} \in[0,1]$ and $\sum_{i=1}^{\tau} w_{i}=1$. If

$$
q-\operatorname{RODHFWBM} M_{\tau}^{s, t}\left(d_{1}, d_{2}, \ldots, d_{\tau}\right)=\left(\frac{1}{\tau(\tau-1)}\left(\oplus_{\substack{i, j=1 \\ i \neq j}}^{\tau}\left(w_{i} d_{i}\right)^{s} \otimes\left(w_{j} d_{j}\right)^{t}\right)\right)^{\frac{1}{s+t}} s, t>0
$$

Then we say $q-R O D H F W B M_{\tau}^{s, t}$ the q-rung orthopair dual hesitant fuzzy weighted Bonferroni mean operator.

Theorem 2. Let $d_{j}=\left(h_{j}, g_{j}\right)(j=1,2, \ldots, \tau)$ be an assortment of q-RODHFNs. The outcome value by using q-RODHFWBM operators is again a q-RODHFN, as shown below.

$$
\begin{aligned}
& q-\operatorname{RODHFWBM} M_{\tau}^{s, t}\left(d_{1}, d_{2}, \ldots, d_{\tau}\right)=\left(\frac{1}{\tau(\tau-1)}\left(\bigoplus_{\substack{i, j=1 \\
i \neq j}}^{\tau}\left(w_{i} d_{i}\right)^{s} \otimes\left(w_{j} d_{j}\right)^{t}\right)\right)^{\frac{1}{s+t}} \\
& =\cup_{\rho_{i} \in h_{i}, \rho_{j} \in h_{j}, \kappa_{i} \in g_{i}, \kappa_{j} \in g_{j}}\left\{\left(\sqrt[q]{1-\prod_{\substack{i, j=1 \\
i \neq j}}^{\tau}\left(1-\left(1-\left(1-\rho_{i}^{q}\right)^{w_{i}}\right)^{s}\left(1-\left(1-\rho_{j}^{q}\right)^{w_{j}}\right)^{t}\right)^{\frac{1}{\tau(\tau-1)}} \frac{1}{s+t}}\right.\right. \\
& \left.\sqrt[q]{1-\left(1-\prod_{\substack{i, j=1 \\
i \neq j}}^{\tau}\left(1-\left(1-\kappa_{i}^{w_{i} q}\right)^{s}\left(1-\kappa_{j}^{w_{j} q}\right)^{t}\right) \frac{1}{\tau(\tau-1)}\right) \frac{1}{s+t}}\right\}
\end{aligned}
$$

Proof. According to definition (2.8), we can obtain the following identities

$$
\begin{gathered}
w_{i} d_{i}=\cup_{\rho_{i} \in h_{i}, \kappa_{i} \in g_{i}}\left\{\left\{\sqrt[q]{1-\left(1-\rho_{i}^{q}\right)^{w_{i}}}\right\},\left\{\kappa_{i}^{w_{i}}\right\}\right\} \\
w_{j} d_{j}=\cup_{\rho_{j} \in h_{j}, \kappa_{j} \in g_{j}}\left\{\left\{\sqrt[q]{1-\left(1-\rho_{j}^{q}\right)^{w_{j}}}\right\},\left\{\kappa_{j}^{w_{j}}\right\}\right\} \\
\left(w_{i} d_{i}\right)^{s}=\cup_{\rho_{i} \in h_{i}, \kappa_{i} \in g_{i}}\left\{\left\{\left(\sqrt[q]{1-\left(1-\rho_{i}^{q}\right)^{w_{i}}}\right)^{s}\right\},\left\{\sqrt[q]{1-\left(1-\kappa_{i}^{w_{i} q}\right)^{s}}\right\}\right\} \\
\left(w_{j} d_{j}\right)^{t}=\cup_{\rho_{j} \in h_{j}, \kappa_{j} \in g_{j}}\left\{\left\{\left(\sqrt[q]{1-\left(1-\rho_{j}^{q}\right)^{w_{j}}}\right)^{t}\right\},\left\{\sqrt[q]{1-\left(1-\kappa_{j}^{w_{j} q}\right)^{t}}\right\}\right\}
\end{gathered}
$$




$$
\begin{aligned}
& \left(w_{i} d_{i}\right)^{s} \otimes\left(w_{j} d_{j}\right)^{t}=\cup_{\rho_{i} \in h_{i}, \kappa_{i} \in g_{i}, \rho_{j} \in h_{j}, \kappa_{j} \in g_{j}} \\
& \left\{\left(\sqrt[q]{1-\left(1-\rho_{i}^{q}\right)^{w_{i}}}\right)^{s}\left(\sqrt[q]{1-\left(1-\rho_{j}^{q}\right)^{w_{j}}}\right)^{t}, \sqrt[q]{1-\left(1-\kappa_{i}^{w_{i} q}\right)^{s}\left(1-\kappa_{j}^{w_{j} q}\right)^{t}}\right\} \\
& \underset{\substack{i, j=1 \\
i \neq j}}{\tau}\left(w_{i} d_{i}\right)^{s} \otimes\left(w_{j} d_{j}\right)^{t}=\cup_{\rho_{i} \in h_{i}, \kappa_{i} \in g_{i}, \rho_{j} \in h_{j}, \kappa_{j} \in g_{j}} \\
& \left\{\sqrt[q]{1-\prod_{\substack{i, j=1 \\
i \neq j}}^{\tau}\left(1-\left(1-\left(1-\rho_{i}^{q}\right)^{w_{i}}\right)^{s}\left(1-\left(1-\rho_{j}^{q}\right)^{w_{j}}\right)^{t}\right)}\right. \\
& \left.\frac{q}{\frac{1}{\prod_{\substack{\tau, j=1 \\
i \neq j}}^{\tau}\left(1-\left(1-\kappa_{i}^{w_{i} q}\right)^{s}\left(1-\kappa_{j}^{w_{j} q}\right)^{t}\right)}}\right\} \\
& \left\{\sqrt[q]{1-\prod_{\substack{i, j=1 \\
i \neq j}}^{\tau}\left(1-\left(1-\left(1-\rho_{i}^{q}\right)^{w_{i}}\right)^{s}\left(1-\left(1-\rho_{j}^{q}\right)^{w_{j}}\right)^{t}\right)^{\frac{1}{\tau(\tau-1)}}},\right. \\
& \left.\left.\left(\sqrt[q]{\prod_{\substack{i, j=1 \\
i \neq j}}^{\tau}\left(1-\left(1-\kappa_{i}^{w_{i} q}\right)^{s}\left(1-\kappa_{j}^{w_{j} q}\right)^{t}\right.}\right)\right)^{\frac{1}{\tau(\tau-1)}}\right\} \\
& \left(\frac{1}{\tau(\tau-1)}\left(\oplus_{\substack{i, j=1 \\
i \neq j}}^{\tau}\left(w_{i} d_{i}\right)^{s} \otimes\left(w_{j} d_{j}\right)^{t}\right)\right)^{\frac{1}{s+t}}=\cup_{\rho_{i} \in h_{i}, \kappa_{i} \in g_{i}, \rho_{j} \in h_{j}, \kappa_{j} \in g_{j}} \\
& \left\{\left(\sqrt[q]{1-\prod_{\substack{i, j=1 \\
i \neq j}}^{\tau}\left(1-\left(1-\left(1-\rho_{i}^{q}\right)^{w_{i}}\right)^{s}\left(1-\left(1-\rho_{j}^{q}\right)^{w_{j}}\right)^{t}\right)^{\frac{1}{\tau(\tau-1)}}} \frac{1}{s+t},\right.\right. \\
& \left.\left.\sqrt[q]{1-\left(1-\prod_{\substack{i, j=1 \\
i \neq j}}^{\tau}\left(1-\left(1-\kappa_{i}^{w_{i} q}\right)^{s}\left(1-\kappa_{j}^{w_{j} q}\right)^{t}\right)\right.} \frac{1}{\tau(\tau-1)}\right) \frac{1}{s+t}\right\}
\end{aligned}
$$

Hence, Eq.16 is preserved.

Now, we must prove that Eq.16 is a q-RODHFN. For this we should prove these two criteria:

1. $0 \leq \rho, \kappa \leq 1$

2. $\cup_{\rho \in h}(\max (\rho))^{q}+\cup_{\kappa \in g}(\max (\kappa))^{q} \leq 1$.

Let

$$
\begin{gathered}
\rho=\left(\sqrt[q]{1-\prod_{\substack{i, j=1 \\
i \neq j}}^{\tau}\left(1-\left(1-\left(1-\rho_{i}^{q}\right)^{w_{i}}\right)^{s}\left(1-\left(1-\rho_{j}^{q}\right)^{w_{j}}\right)^{t}\right)^{\frac{1}{\tau(\tau-1)}}}\right)^{\frac{1}{s+t}} \\
\left.\kappa=\sqrt[q]{1-\left(1-\prod_{\substack{i, j=1 \\
i \neq j}}^{\tau}\left(1-\left(1-\kappa_{i}^{w_{i} q}\right)^{s}\left(1-\kappa_{j}^{w_{j} q}\right)^{t}\right.\right.}\right)^{\left.\frac{1}{\tau(\tau-1)}\right)^{\frac{1}{s+t}}}
\end{gathered}
$$

Proof : 1 . Since $0 \leq \rho_{j} \leq 1$, we get $0 \leq\left(1-\rho_{j}^{q}\right)^{w_{j}} \leq 1$ and

$$
0 \leq 1-\prod_{\substack{i, j=1 \\ i \neq j}}^{\tau}\left(1-\left(1-\left(1-\rho_{j}^{q}\right)^{w_{j}}\right)^{s}\left(1-\left(1-\rho_{j}^{q}\right)^{w_{j}}\right)^{t}\right)^{\frac{1}{\tau(\tau-1)}} \leq 1
$$


Then,

$$
0 \leq\left(\sqrt[q]{\left.1-\prod_{\substack{i, j=1 \\ i \neq j}}^{\tau}\left(1-\left(1-\left(1-\rho_{i}^{q}\right)^{w_{i}}\right)^{s}\left(1-\left(1-\rho_{j}^{q}\right)^{w_{j}}\right)^{t}\right)^{\frac{1}{\tau(\tau-1)}}\right)^{\frac{1}{s+t}}} \leq 1\right.
$$

That means $0 \leq \rho \leq 1$, on same lines, we can find $0 \leq \kappa \leq 1$. Hence 1 . is preserved.

For $(\max (\rho))^{q}+(\max (\kappa))^{q} \leq 1$, we have this expression

$$
\begin{aligned}
& \cup_{\rho \in h}(\max (\rho))^{q}+\cup_{\kappa \in g}(\max (\kappa))^{q} \\
& =\left(1-\prod_{\substack{i, j=1 \\
i \neq j}}^{\tau}\left(1-\left(1-\left(1-\rho_{i}^{q}\right)^{w_{i}}\right)^{s}\left(1-\left(1-\rho_{j}^{q}\right)^{w_{j}}\right)^{t}\right)^{\frac{1}{\tau(\tau-1)}}\right)^{\frac{1}{s+t}} \\
& +1-\left(1-\prod_{\substack{i \neq j=1 \\
i \neq j}}^{\tau}\left(1-\left(1-\kappa_{i}^{w_{i} q}\right)^{s}\left(1-\kappa_{j}^{w_{j} q}\right)^{t}\right)^{\frac{1}{\tau(\tau-1)}}\right)^{\frac{1}{s+t}} \\
& \leq\left(1-\prod_{\substack{i, j=1 \\
i \neq j}}^{\tau}\left(1-\left(1-\left(1-\rho_{i}^{q}\right)^{w_{i}}\right)^{s}\left(1-\left(1-\rho_{j}^{q}\right)^{w_{j}}\right)^{t}\right)^{\frac{1}{\tau(\tau-1)}}\right)^{\frac{1}{s+t}} \\
& +1-\left(1-\prod_{\substack{i, j=1 \\
i \neq j}}^{\tau}\left(1-\left(1-\left(1-\rho_{i}^{q}\right)^{w_{i}}\right)^{s}\left(1-\left(1-\rho_{j}^{q}\right)^{w_{j}}\right)^{t}\right)^{\frac{1}{\tau(\tau-1)}}\right)^{\frac{1}{s+t}}=1
\end{aligned}
$$

So 2. is preserved also.

Example 2.2. Let $d_{1}=\{\{0.3,0.4\},\{0.5\}\}, d_{2}=\{\{0.7\},\{0.1,0.2,0.6\}\}$, and $d_{3}=\{\{0.6\},\{0.3\}\}$ be three q-RODHFNs, and let $s=1, t=1$ and $q=3$ then using Eq.(16), we get for the membership (favorable) function $\rho$, the final outcomes are given as below.

Alike, we can find $\rho_{2}=q-\operatorname{RODHFBM}(0.4,0.7,0.6)=0.4173$, and $\rho=\{0.4032,0.4173\}$. For the non-membership (unfavorable) function $\kappa$, the final results are shown here. Alike, the results of $\kappa_{2}$, and $\kappa_{3}$, are $\kappa_{2}=\mathrm{q}-\mathrm{RODHFBM}(0.5,0.2$, $0.3)=0.4569, \kappa_{3}=\mathrm{q}-\operatorname{RODHFBM}(0.5,0.6,0.3)=0.5970$, so we have $\kappa=\{0.6799,0.4569,0.5970\}$. Therefore,

$$
q-\operatorname{RODHFBM}\left(d_{1}, d_{2}, d_{3}\right)=\{\{0.4032,0.4173\},\{0.6799,0.4569,0.5970\}\}
$$

For some particular values of parameter $q$, the important cases of q-RODHFWBM operator are discussed here.

1) For parameter $q$, there exist following important cases

Remark 7. When $q=1$, the q-RODHFWBM operator will turn into dual hesitant fuzzy weighted BM (DHFWBM) operator as shown below:

$$
\begin{aligned}
& \operatorname{DHFWBM}_{\tau}^{s, t}\left(d_{1}, d_{2}, \ldots, d_{\tau}\right)=\left(\frac{1}{\tau(\tau-1)}\left(\oplus_{\substack{i, j=1 \\
i \neq j}}^{\tau}\left(w_{i} d_{i}\right)^{s} \otimes\left(w_{j} d_{j}\right)^{t}\right)\right)^{\frac{1}{s+t}} \\
& =\cup_{\rho_{i} \in h_{i}, \rho_{j} \in h_{j}, \kappa_{i} \in g_{i}, \kappa_{j} \in g_{j}}\left\{\left(1-\prod_{\substack{i, j=1 \\
i \neq j}}^{\tau}\left(1-\left(1-\left(1-\rho_{i}\right)^{w_{i}}\right)^{s}\left(1-\left(1-\rho_{j}\right)^{w_{j}}\right)^{t}\right)^{\left.\frac{1}{\tau(\tau-1)}\right)} \frac{1}{s+t},\right.\right. \\
& \left.1-\left(1-\prod_{\substack{i, j=1 \\
i \neq j}}^{\tau}\left(1-\left(1-\kappa_{i}^{w_{i}}\right)^{s}\left(1-\kappa_{j}^{w_{j}}\right)^{t}\right) \frac{1}{\tau(\tau-1)}\right) \frac{1}{s+t}\right\}
\end{aligned}
$$


Remark 8. When $q=2$, the q-RODHFWBM operator will turn into dual hesitant Pythagorean fuzzy weighted BM (DHPFWBM) as shown below:

$$
\begin{aligned}
& \operatorname{DHPFWBM} M_{w}^{s, t}\left(d_{1}, d_{2}, \ldots, d_{\tau}\right)=\left(\frac{1}{\tau(\tau-1)}\left(\bigoplus_{\substack{i, j=1 \\
i \neq j}}^{\tau}\left(w_{i} d_{i}\right)^{s} \otimes\left(w_{j} d_{j}\right)^{t}\right)\right)^{\frac{1}{s+t}} \\
& =\cup_{\rho_{i} \in h_{i}, \rho_{j} \in h_{j}, \kappa_{i} \in g_{i}, \kappa_{j} \in g_{j}}\left\{\left(\sqrt{1-\prod_{\substack{i, j=1 \\
i \neq j}}^{\tau}\left(1-\left(1-\left(1-\rho_{i}^{2}\right)^{w_{i}}\right)^{s}\left(1-\left(1-\rho_{j}^{2}\right)^{w_{j}}\right)^{t}\right)^{\overline{\tau(\tau-1)}}}\right)^{\frac{1}{s+t}},\right. \\
& \left.\sqrt{1-\left(1-\prod_{\substack{i, j=1 \\
i \neq j}}^{\tau}\left(1-\left(1-\kappa_{i}^{2 w_{i}}\right)^{s}\left(1-\kappa_{j}^{2 w_{j}}\right)^{t}\right) \frac{1}{\tau(\tau-1)}\right)^{\frac{1}{s+t}}}\right\}
\end{aligned}
$$

2) For parameter $s$ and $t$, there exist some important cases.

Remark 9. When $t \rightarrow 0$, the q-RODHFWBM will turn into the q-rung orthopair dual hesitant fuzzy weighted arithmetic mean (q-RODHFWAM) as shown below:

$$
\begin{aligned}
& q-\operatorname{RODHFWAM} M_{\tau}^{s, 0}\left(d_{1}, d_{2}, \ldots, d_{\tau}\right)=\left(\frac{1}{\tau(\tau-1)}\left(\oplus_{\substack{i, j=1 \\
i \neq j}}^{\tau}\left(w_{i} d_{i}\right)^{s}\right)\right)^{\frac{1}{s}} \\
& =\cup_{\rho_{i} \in h_{i}, \rho_{j} \in h_{j}, \kappa_{i} \in g_{i}, \kappa_{j} \in g_{j}}\left\{\left(\sqrt[q]{1-\prod_{\substack{i, j=1 \\
i \neq j}}^{\tau}\left(1-\left(1-\left(1-\rho_{i}^{q}\right)^{w_{i}}\right)^{s}\right) \frac{1}{\tau}} \frac{1}{s}\right.\right. \\
& \left.\sqrt[q]{1-\left(1-\prod_{\substack{i, j=1 \\
i \neq j}}^{\tau}\left(1-\left(1-\kappa_{i}^{w_{i} q}\right)^{s}\right) \frac{1}{\tau}\right.} \frac{1}{s}\right\}
\end{aligned}
$$

Remark 10. If $s=2$ and $t \rightarrow 0$, the q-RODHFWBM will turn into the q-rung orthopair dual hesitant fuzzy weighted square mean (q-RODHFWSM) as shown below:

$$
\begin{aligned}
& q-\operatorname{RODHFWSM} M_{\tau}^{2,0}\left(d_{1}, d_{2}, \ldots, d_{\tau}\right)=\left(\frac{1}{\tau(\tau-1)}\left(\oplus_{\substack{i, j=1 \\
i \neq j}}^{\tau}\left(w_{i} d_{i}\right)^{2} \otimes\left(w_{j} d_{j}\right)^{0}\right)\right)^{\frac{1}{2}} \\
& =\cup_{\rho_{i} \in h_{i}, \rho_{j} \in h_{j}, \kappa_{i} \in g_{i}, \kappa_{j} \in g_{j}}\left\{\left(\sqrt[q]{1-\prod_{\substack{i, j=1 \\
i \neq j}}^{\tau}\left(1-\left(1-\left(1-\rho_{i}^{q}\right)^{w_{i}}\right)^{2}\right)} \frac{1}{\tau}\right)^{\frac{1}{2}},\right. \\
& \sqrt[q]{1-\left(1-\prod_{\substack{i, j=1 \\
i \neq j}}^{\tau}\left(1-\left(1-\kappa_{i}^{w_{i} q}\right)^{2}\right)^{\frac{1}{\tau}}\right)^{\frac{1}{2}}}
\end{aligned}
$$


Remark 11. If $s=1$ and $t \rightarrow 0$, the q-RODHFWBM will turn into the q-rung orthopair dual hesitant fuzzy weighted geometric mean (q-RODHFWGM) operator as shown below:

$$
\begin{aligned}
& q-\operatorname{RODHFWA} A_{\tau}^{1,0}\left(d_{1}, d_{2}, \ldots, d_{\tau}\right)=\left(\frac{1}{\tau(\tau-1)}\left(\oplus_{\substack{i, j=1 \\
i \neq j}}^{\tau}\left(w_{i} d_{i}\right)^{1} \otimes\left(w_{j} d_{j}\right)^{0}\right)\right) \\
& =\cup_{\rho_{i} \in h_{i}, \rho_{j} \in h_{j}, \kappa_{i} \in g_{i}, \kappa_{j} \in g_{j}}\left\{\sqrt[q]{1-\prod_{\substack{i, j=1 \\
i \neq j}}^{\tau}\left(\left(1-\rho_{i}^{q}\right)^{w_{i}}\right)} \frac{1}{\tau} \sqrt[q]{\prod_{\substack{i, j=1 \\
i \neq j}}^{\tau}\left(\kappa_{i}^{w_{i} q}\right) \frac{1}{\tau}}\right\}
\end{aligned}
$$

Remark 12. When $s=1$ and $t=1$, the q-RODHFWBM will turn into the q-rung orthopair dual hesitant fuzzy weighted interrelated square mean (q-RODHFWISM) operator as shown below:

$$
\begin{aligned}
& q-\operatorname{RODHFWISM}_{\tau}^{1,1}\left(d_{1}, d_{2}, \ldots, d_{\tau}\right)=\left(\frac{1}{\tau(\tau-1)}\left(\bigoplus_{\substack{i, j=1 \\
i \neq j}}^{\tau}\left(w_{i} d_{i}\right)^{1} \otimes\left(w_{j} d_{j}\right)^{1}\right)\right)^{\frac{1}{2}} \\
& =\cup_{\rho_{i} \in h_{i}, \rho_{j} \in h_{j}, \kappa_{i} \in g_{i}, \kappa_{j} \in g_{j}}\left\{\left(\sqrt[q]{1-\prod_{\substack{i, j=1 \\
i \neq j}}^{\tau}\left(1-\left(1-\left(1-\rho_{i}^{q}\right)^{w_{i}}\right)^{1}\left(1-\left(1-\rho_{j}^{q}\right)^{w_{j}}\right)^{1}\right)^{\frac{1}{\tau(\tau-1)}}} \frac{1}{\frac{1}{2}},\right.\right. \\
& \left.\sqrt[q]{1-\left(1-\prod_{\substack{i, j=1 \\
i \neq j}}^{\tau}\left(1-\left(1-\kappa_{i}^{w_{i} q}\right)^{1}\left(1-\kappa_{j}^{w_{j} q}\right)^{1}\right) \frac{1}{\tau(\tau-1)}\right)^{\frac{1}{2}}}\right\}
\end{aligned}
$$

\subsection{The q-RODHFDBM OPERATOR}

Now, we establish the dual BM (DBM) combining both the BM and dual operation.

Definition 2.12. Let $s, t \geq 0$ and $a_{i}(i=1,2, \ldots, \tau)$ be an assortment of nonnegative real numbers. If

$$
D B M^{s, t}\left(a_{1}, a_{2}, \ldots, a_{\tau}\right)=\frac{1}{s+t}\left(\prod_{\substack{i, j=1 \\ i \neq j}}^{\tau}\left(s a_{i}+t a_{j}\right)\right)^{\frac{1}{\tau(\tau-1)}}
$$

Then we call $\mathrm{DBM}^{s, t}$ the dual BM (DBM) operator.

Now, we shall introduce the DBM operator for q-RODHFNs as follows.

Definition 2.13. Let $s, t>0$ and $d_{j}=\left(h_{j}, g_{j}\right)(j=1,2, \ldots, \tau)$ be a set of q-RODHFNs. If

$$
q-\operatorname{RODHFDBM} M^{s, t}\left(d_{1}, d_{2}, \ldots, d_{\tau}\right)=\frac{1}{s+t}\left(\otimes_{\substack{i, j=1 \\ i \neq j}}^{\tau}\left(s d_{i} \oplus t d_{j}\right)\right)^{\frac{1}{\tau(\tau-1)}}
$$

Then the name q-RODHFDBM ${ }^{s, t}$ stands for the q-rung orthopair dual hesitant fuzzy dual Bonferroni mean operator. 
Theorem 3. Let $d_{j}=\left(h_{j}, g_{j}\right)(j=1,2, \ldots, \tau)$ be an assortment of q-RODHFNs. The resulted value by using q-RODHFDBM operator is again a q-RODHFN where as Eq.26, as shown here.

$$
\begin{aligned}
& q-\operatorname{RODHFDBM} M^{s, t}\left(d_{1}, d_{2}, \ldots, d_{\tau}\right)=\frac{1}{s+t}\left(\otimes_{\substack{i, j=1 \\
i \neq j}}^{\tau}\left(s d_{i} \oplus t d_{j}\right)\right)^{\frac{1}{\tau(\tau-1)}} \\
& =\cup_{\rho_{i} \in h_{i}, \kappa_{i} \in g_{i}, \rho_{j} \in h_{j}, \kappa_{j} \in g_{j}}\left\{\sqrt[q]{1-\left(1-\prod_{\substack{i, j=1 \\
i \neq j}}^{\tau}\left(1-\left(1-\rho_{i}^{q}\right)^{s}\left(1-\rho_{j}^{q}\right)^{t}\right)^{\frac{1}{\tau(\tau-1)}}\right) \frac{1}{s+t}},\right. \\
& \left.\left(\sqrt[q]{1-\prod_{\substack{i, j=1 \\
i \neq j}}^{\tau}\left(1-\left(\kappa_{i}^{s} \kappa_{j}^{t}\right)^{q}\right)^{\frac{1}{\tau(\tau-1)}}}\right) \frac{1}{s+t}\right\}
\end{aligned}
$$

Based on operations (1)-(4) of the q-RODHFNs stated in Section 2, we can drive the following result.

Proof. From definition (2.8), the proof follows easily.

Example 2.3. Let $d_{1}=\{\{0.3,0.4\},\{0.5\}\}, d_{2}=\{\{0.7\},\{0.1,0.2,0.6\}\}$, and $d_{3}=\{\{0.6\},\{0.3\}\}$ be three q-RODHFNs, and let $s=1, t=1$ and $q=3$ then using Eq.(26), we get for the membership (favorable) function $\rho$, the ultimate outcomes are as below.

On same lines, we have found $\rho_{2}=q-\operatorname{RODHFDBM}(0.4,0.7,0.6)=0.5743$, and $\rho=\{0.5763,0.5743\}$. For the nonmembership (unfavorable) function $\kappa$, the ultimate outcomes are shown as . Alike, the results of $\kappa_{2}$, and $\kappa_{3}$, are $\kappa_{2}=\mathrm{q}-$ $\operatorname{RODHFBM}(0.5,0.2,0.3)=0.3404, \kappa_{3}=\mathrm{q}-\operatorname{RODHFDBM}(0.5,0.6,0.3)=0.5745$, so we can list $\kappa=\{0.3303,0.3404,0.5745\}$.

Therefore,

$$
q-\operatorname{RODHFBM}\left(d_{1}, d_{2}, d_{3}\right)=\{\{0.5763,0.5743\},\{0.3303,0.3404,0.5745\}\}
$$

By adjusting the estimations of parameter $s, t$ and $q$, some unique instances of q-RODHFBM operator are given as pursues.

(1) For parameter q, there arise some important cases

Remark 13. When $q=1$, the q-RODHFDBM operator will turn into dual hesitant fuzzy DBM (DHFDBM) operator as shown below:

$$
\begin{aligned}
& \operatorname{DHFDBM}^{s, t}\left(d_{1}, d_{2}, \ldots, d_{\tau}\right)=\frac{1}{s+t}\left(\otimes_{i, j=1_{i \neq j}}^{\tau}\left(s d_{i} \oplus t d_{j}\right)\right)^{\frac{1}{\tau(\tau-1)}} \\
& =\cup_{\rho_{i} \in h_{i}, \kappa_{i} \in g_{i}, \rho_{j} \in h_{j}, \kappa_{j} \in g_{j}}\left\{1-\left(1-\prod_{\substack{i, j=1 \\
i \neq j}}^{\tau}\left(1-\left(1-\rho_{i}^{q}\right)^{s}\left(1-\rho_{j}^{q}\right)^{t}\right) \frac{1}{\tau(\tau-1)} \frac{1}{s+t},\right.\right. \\
& \left.\left(1-\prod_{\substack{i, j=1 \\
i \neq j}}^{\tau}\left(1-\left(\kappa_{i}^{s} \kappa_{j}^{t}\right)^{q}\right)^{\frac{1}{\tau(\tau-1)}}\right) \frac{1}{s+t}\right\}
\end{aligned}
$$


Remark 14. When $q=2$, the q-RODHFDBM operator will turn into dual hesitant Pythagorean fuzzy DBM (DHPFDBM) which can be presented in,

$$
\begin{aligned}
& \operatorname{DHPFDBM} M^{s, t}\left(d_{1}, d_{2}, \ldots, d_{\tau}\right)=\frac{1}{s+t}\left(\otimes_{i, j=1_{i \neq j}}^{\tau}\left(s d_{i} \oplus t d_{j}\right)\right)^{\frac{1}{\tau(\tau-1)}} \\
& =\cup_{\rho_{i} \in h_{i}, \kappa_{i} \in g_{i}, \rho_{j} \in h_{j}, \kappa_{j} \in g_{j}}\left\{\sqrt{1-\left(1-\prod_{\substack{i, j=1 \\
i \neq j}}^{\tau}\left(1-\left(1-\rho_{i}^{2}\right)^{s}\left(1-\rho_{j}^{2}\right)^{t}\right)^{\frac{1}{\tau(\tau-1)}} \frac{1}{s+t}\right.},\right. \\
& \left(\sqrt{1-\prod_{\substack{i, j=1 \\
i \neq j}}^{\tau}\left(1-\left(\kappa_{i}^{s} \kappa_{j}^{t}\right)^{2}\right)^{\frac{1}{\tau(\tau-1)}}} \frac{1}{s+t}\right\}
\end{aligned}
$$

(2) For parameter $s$ and $t$, there exist these important cases.

Remark 15. If $t \rightarrow 0$, then q-RODHFDBM will turn into the q-rung orthopair dual hesitant fuzzy dual arithmetic mean (q-RODHFDAM) operator as shown below:

$$
\begin{aligned}
& q-\operatorname{RODHFDAM} M^{s, 0}\left(d_{1}, d_{2}, \ldots, d_{\tau}\right)=\frac{1}{s}\left(\left(\otimes_{i=1}^{\tau}\left(s d_{i}\right)\right)^{\frac{1}{\tau(\tau-1)}}\right) \\
& =\cup_{\rho_{i} \in h_{i}, \kappa_{i} \in g_{i}}\left\{\sqrt[q]{1-\left(1-\prod_{i=1}^{\tau}\left(1-\left(1-\rho_{i}^{q}\right)^{s}\right) \frac{1}{\tau}\right.} \frac{1}{s},\left(\sqrt[q]{1-\prod_{i=1}^{\tau}\left(1-\left(\kappa_{i}^{s}\right)^{q}\right) \frac{1}{\tau}}\right)^{\frac{1}{s}}\right\}
\end{aligned}
$$

Remark 16. If $s=2$ and $t \rightarrow 0$, then the q-RODHFDBM will turn into the q-rung orthopair dual hesitant fuzzy dual square mean (q-RODHFDSM) as shown below:

$$
\begin{aligned}
& q-\text { RODHROFAM }{ }^{2,0}\left(d_{1}, d_{2}, \ldots, d_{\tau}\right)=\frac{1}{2}\left(\left(\otimes_{i=1}^{\tau}\left(s d_{i}\right)\right)^{\frac{1}{\tau(\tau-1)}}\right) \\
& \left.=\cup_{\rho_{i} \in h_{i}, \kappa_{i} \in g_{i}}\left\{\sqrt[q]{1-\left(1-\prod_{i=1}^{\tau}\left(1-\left(1-\rho_{i}^{q}\right)^{2}\right)^{\frac{1}{\tau}}\right.}\right)^{\frac{1}{2}},\left(\sqrt[q]{1-\prod_{i=1}^{\tau}\left(1-\left(\kappa_{i}^{2}\right)^{q}\right) \frac{1}{\tau}}\right)^{\frac{1}{2}}\right\}
\end{aligned}
$$

Remark 17. If $s=1$ and $t \rightarrow 0$, then the q-RODHFDBM will turn into the q-rung orthopair dual hesitant fuzzy dual geometric mean (q-RODHFDGM) operator as shown below:

$$
\begin{aligned}
& q-\operatorname{RODHFAM} M^{1,0}\left(d_{1}, d_{2}, \ldots, d_{\tau}\right)=\frac{1}{1}\left(\left(\otimes_{i=1}^{\tau}\left(s d_{i}\right)\right)^{\frac{1}{\tau(\tau-1)}}\right) \\
& =\cup_{\rho_{i} \in h_{i}, \kappa_{i} \in g_{i}, \rho_{j} \in h_{j}}\left\{\sqrt[q]{\prod_{i=1}^{\tau}\left(\rho_{i}^{q}\right) \frac{1}{\tau}}, \sqrt[q]{1-\prod_{i=1}^{\tau}\left(1-\left(\kappa_{i}\right)^{q}\right)^{\frac{1}{\tau}}}\right\}
\end{aligned}
$$


Remark 18. If $s=1$ and $t=1$, then the q-RODHFDBM will turn into the q-rung orthopair dual hesitant fuzzy interrelated square mean (q-RODHFDISM) operator as shown below:

$$
\begin{aligned}
& q-\operatorname{RODHFDISM} M^{1,1}\left(d_{1}, d_{2}, \ldots, d_{\tau}\right)=\frac{1}{2}\left(\otimes_{\substack{i, j=1 \\
i \neq j}}^{\tau}\left(d_{i} \oplus d_{j}\right)\right)^{\frac{1}{\tau(\tau-1)}} \\
& =\cup_{\rho_{i} \in h_{i}, \kappa_{i} \in g_{i}, \rho_{j} \in h_{j}, \kappa_{j} \in g_{j}}\left\{\sqrt[q]{1-\left(1-\prod_{\substack{i, j=1 \\
i \neq j}}^{\tau}\left(1-\left(1-\rho_{i}^{q}\right)\left(1-\rho_{j}^{q}\right)\right)^{\frac{1}{\tau(\tau-1)}}\right)^{\frac{1}{2}}},\right. \\
& \left.\left(\sqrt[q]{1-\prod_{\substack{i, j=1 \\
i \neq j}}^{\tau}\left(1-\left(\kappa_{i} \kappa_{j}\right)^{q}\right)^{\frac{1}{\tau(\tau-1)}}}\right)^{\frac{1}{2}}\right\}
\end{aligned}
$$

\subsection{THE q-RODHFWDBM OPERATOR}

In actual MADM, it's good to assign weights to each attribute. In this segment, we shall explore the q-rung orthopair dual hesitant fuzzy weighted dual Bonferroni mean (q-RODHFWDBM) operator as pursues.

Definition 2.14. Let $s, t>0$ and $a_{i}(i=1,2, \ldots, \tau)$ be a set of nonnegative real numbers. If

$$
D B M^{s, t}\left(a_{1}, a_{2}, \ldots, a_{\tau}\right)=\frac{1}{s+t}\left(\prod_{\substack{i, j=1 \\ i \neq j}}^{\tau}\left(s a_{i}^{w_{i}}+t a_{j}^{w_{j}}\right)\right)^{\frac{1}{\tau(\tau-1)}}
$$

Then we call $\mathrm{DBM}^{s, t}$ the dual BM (DBM) operator.

Now, we will establish the DBM operator for q-RODHFNs as follows.

Definition 2.15. Let $s, t>0$ and $d_{j}=\left(h_{j}, g_{j}\right)(j=1,2, \ldots, \tau)$ be an assortment of q-RODHFNs. If

$$
q-\operatorname{RODHFWDBM} M^{s, t}\left(d_{1}, d_{2}, \ldots, d_{\tau}\right)=\frac{1}{s+t}\left(\otimes_{\substack{i, j=1 \\ i \neq j}}^{\tau}\left(s d_{i}^{w_{i}} \oplus t d_{j}^{w_{j}}\right)\right)^{\frac{1}{\tau(\tau-1)}}
$$

Then q-RODHFWDBM ${ }^{s, t}$ stands for the q-rung orthopair dual hesitant fuzzy weighted dual Bonferroni mean operator.

Theorem 4. Let $s, t>0$ and $d_{j}=\left(h_{j}, g_{j}\right)(j=1,2, \ldots, \tau)$ be an assortment of q-RODHFNs. The aggregated result after utilizing q-RODHFWDBM operators is again a q-RODHFN where as Eq.34, as shown here.

$$
\begin{aligned}
& q-\operatorname{RODHFWDBM} M^{s, t}\left(d_{1}, d_{2}, \ldots, d_{\tau}\right)=\frac{1}{s+t}\left(\otimes_{\substack{i, j=1 \\
i \neq j}}^{\tau}\left(s d_{i}^{w_{i}} \oplus t d_{j}^{w_{j}}\right)\right)^{\frac{1}{\tau(\tau-1)}} \\
& =\cup_{\rho_{i} \in h_{i}, \kappa_{i} \in g_{i}, \rho_{j} \in h_{j}, \kappa_{j} \in g_{j}}\left\{\sqrt[q]{1-\left(1-\prod_{\substack{i, j=1 \\
i \neq j}}^{\tau}\left(1-\left(1-\rho_{i}^{w_{i} q}\right)^{s}\left(1-\rho_{j}^{w_{j} q}\right)^{t}\right)^{\frac{1}{\tau(\tau-1)}} \frac{1}{s+t}\right.},\right. \\
& \left.\left(\sqrt[q]{1-\prod_{\substack{i, j=1 \\
i \neq j}}^{\tau}\left(1-\left(1-\left(1-\kappa_{i}^{q}\right)^{w_{i}}\right)^{s}\left(1-\left(1-\kappa_{j}^{q}\right)^{w_{j}}\right)^{t}\right)^{\frac{1}{\tau(\tau-1)}}}\right)^{\frac{1}{s+t}}\right\}
\end{aligned}
$$


Proof. From definition (2.8), we can obtain the following identities

$$
\begin{aligned}
& d_{i}^{w_{i}}=\cup_{\rho_{i} \in h_{i}, \kappa_{i} \in g_{i}}\left\{\rho_{i}^{w_{i}}, \sqrt[q]{1-\left(1-\kappa_{i}^{q}\right)^{w_{i}}}\right\} \\
& s d_{i}^{w_{i}}=\cup_{\rho_{i} \in h_{i}, \kappa_{i} \in g_{i}}\left\{\sqrt[q]{1-\left(1-\rho_{i}^{w_{i} q}\right)^{s}},\left(\sqrt[q]{1-\left(1-\kappa_{i}^{q}\right)^{w_{i}}}\right)^{s}\right\} \\
& d_{j}^{w_{j}}=\cup_{\rho_{j} \in h_{j}, \kappa_{j} \in g_{j}}\left\{\rho_{j}^{w_{j}}, \sqrt[q]{1-\left(1-\kappa_{j}^{q}\right)^{w_{j}}}\right\} \\
& t d_{j}^{w_{j}}=\cup_{\rho_{j} \in h_{j}, \kappa_{j} \in g_{j}}\left\{\sqrt[q]{1-\left(1-\rho_{j}^{w_{j} q}\right)^{t}},\left(\sqrt[q]{1-\left(1-\kappa_{j}^{q}\right)^{w_{j}}}\right)^{t}\right\} \\
& s d_{i}^{w_{i}} \oplus t d_{j}^{w_{j}}=\cup_{\rho_{i} \in h_{i}, \kappa_{i} \in g_{i}, \rho_{j} \in h_{j}, \kappa_{j} \in g_{j}} \\
& \left\{\sqrt[q]{1-\left(1-\rho_{i}^{w_{i} q}\right)^{s}\left(1-\rho_{j}^{w_{j} q}\right)^{t}},\left(\sqrt[q]{1-\left(1-\kappa_{i}^{q}\right)^{w_{i}}}\right)^{s}\left(\sqrt[q]{1-\left(1-\kappa_{j}^{q}\right)^{w_{j}}}\right)^{t}\right\} \\
& s d_{j}^{w_{j}} \oplus t d_{i}^{w_{i}}=\cup_{\rho_{i} \in h_{i}, \kappa_{i} \in g_{i}, \rho_{j} \in h_{j}, \kappa_{j} \in g_{j}} \\
& \left\{\sqrt[q]{1-\left(1-\rho_{j}^{w_{j} q}\right)^{s}\left(1-\rho_{i}^{w_{i} q}\right)^{t}},\left(\sqrt[q]{1-\left(1-\kappa_{j}^{q}\right)^{w_{j}}}\right)^{s}\left(\sqrt[q]{1-\left(1-\kappa_{i}^{q}\right)^{w_{i}}}\right)^{t}\right\} \\
& \otimes_{\substack{i, j=1 \\
i \neq j}}^{\tau}\left(s d_{i}^{w_{i}} \oplus t d_{j}^{w_{j}}\right)=\cup_{\rho_{i} \in h_{i}, \kappa_{i} \in g_{i}, \rho_{j} \in h_{j}, \kappa_{j} \in g_{j}} \\
& \left\{\sqrt[q]{\prod_{\substack{i, j=1 \\
i \neq j}}^{\tau}\left(1-\left(1-\rho_{i}^{w_{i} q}\right)^{s}\left(1-\rho_{j}^{w_{j} q}\right)^{t}\right)},\right. \\
& \left.\sqrt[q]{1-\prod_{\substack{i, j=1 \\
i \neq j}}^{\tau}\left(1-\left(1-\left(1-\kappa_{i}^{q}\right)^{w_{i}}\right)^{s}\left(1-\left(1-\kappa_{j}^{q}\right)^{w_{j}}\right)^{t}\right)}\right\} \\
& \left(\otimes_{\substack{i, j=1 \\
i \neq j}}^{\tau}\left(s d_{i}^{w_{i}} \oplus t d_{j}^{w_{j}}\right)\right)^{\frac{1}{\tau(\tau-1)}}=\cup_{\rho_{i} \in h_{i}, \kappa_{i} \in g_{i}, \rho_{j} \in h_{j}, \kappa_{j} \in g_{j}} \\
& \left\{\left(\sqrt[q]{\prod_{\substack{i, j=1 \\
i \neq j}}^{\tau}\left(1-\left(1-\rho_{i}^{w_{i} q}\right)^{s}\left(1-\rho_{j}^{w_{j} q}\right)^{t}\right)}\right)^{\frac{1}{\tau(\tau-1)}},\right. \\
& \left.\sqrt[q]{1-\prod_{\substack{i, j=1 \\
i \neq j}}^{\tau}\left(1-\left(1-\left(1-\kappa_{i}^{q}\right)^{w_{i}}\right)^{s}\left(1-\left(1-\kappa_{j}^{q}\right)^{w_{j}}\right)^{t}\right)^{\frac{1}{\tau(\tau-1)}}}\right\} \\
& \frac{1}{s+t}\left(\otimes_{\substack{i, j=1 \\
i \neq j}}^{\tau}\left(s d_{i}^{w_{i}} \oplus t d_{j}^{w_{j}}\right)^{\frac{1}{\tau(\tau-1)}}\right)=\cup_{\rho_{i} \in h_{i}, \kappa_{i} \in g_{i}, \rho_{j} \in h_{j}, \kappa_{j} \in g_{j}} \\
& \left\{\sqrt[q]{1-\left(1-\prod_{\substack{i, j=1 \\
i \neq j}}^{\tau}\left(1-\left(1-\rho_{i}^{w_{i} q}\right)^{s}\left(1-\rho_{j}^{w_{j} q}\right)^{t}\right)^{\frac{1}{\tau(\tau-1)}}\right)^{\frac{1}{s+t}}}\right. \\
& \left.\left(\sqrt[q]{1-\prod_{\substack{i, j=1 \\
i \neq j}}^{\tau}\left(1-\left(1-\left(1-\kappa_{i}^{q}\right)^{w_{i}}\right)^{s}\left(1-\left(1-\kappa_{j}^{q}\right)^{w_{j}}\right)^{t}\right)^{\frac{1}{\tau(\tau-1)}}}\right)^{\frac{1}{s+t}}\right\}
\end{aligned}
$$

Therefore, Eq.36, as shown above.

Hence, Eq.35 is preserved.

Now to show that Eq.35 is a q-RODHFN. It should satisfy these two criteria as follows:

1. $0 \leq \rho, \kappa \leq 1$ 
2. $\cup_{\rho \in h}(\max (\rho))^{q}+\cup_{\kappa \in g}(\max (\kappa))^{q}$

Let

$$
\begin{aligned}
& \rho=\sqrt[q]{1-\left(1-\prod_{\substack{i, j=1 \\
i \neq j}}^{\tau}\left(1-\left(1-\rho_{i}^{w_{i} q}\right)^{s}\left(1-\rho_{j}^{w_{j} q}\right)^{t}\right)^{\frac{1}{\tau(\tau-1)}}\right)^{\frac{1}{s+t}}} \\
& \kappa=\left(\sqrt[q]{1-\prod_{\substack{i, j=1 \\
i \neq j}}^{\tau}\left(1-\left(1-\left(1-\kappa_{i}^{q}\right)^{w_{i}}\right)^{s}\left(1-\left(1-\kappa_{j}^{q}\right)^{w_{j}}\right)^{t}\right)^{\frac{1}{\tau(\tau-1)}}}\right)^{\frac{1}{s+t}}
\end{aligned}
$$

Proof. Since $0 \leq \rho_{j} \leq 1$ we get

$$
\begin{aligned}
& 0 \leq\left(1-\rho_{i}^{w_{i} q}\right)^{s} \leq 1 \\
& 0 \leq \prod_{\substack{i, j=1 \\
i \neq j}}^{\tau}\left(1-\left(1-\rho_{i}^{w_{i} q}\right)^{s}\left(1-\rho_{j}^{w_{j} q}\right)^{t}\right)^{\frac{1}{\tau(\tau-1)}} \leq 1 \\
& 0 \leq\left(1-\prod_{\substack{i, j=1 \\
i \neq j}}^{\tau}\left(1-\left(1-\rho_{i}^{w_{i} q}\right)^{s}\left(1-\rho_{j}^{w_{j} q}\right)^{t}\right)^{\left.\frac{1}{\tau(\tau-1)}\right)^{\frac{1}{s+t}}} \leq 1\right. \\
& 0 \leq \sqrt{1-\left(1-\prod_{\substack{i, j=1 \\
i \neq j}}^{\tau}\left(1-\left(1-\rho_{i}^{w_{i} q}\right)^{s}\left(1-\rho_{j}^{w_{j} q}\right)^{t}\right)^{\frac{1}{\tau(\tau-1)}}\right)^{\frac{1}{s+t}}} \leq 1
\end{aligned}
$$

This states $0 \leq \rho \leq 1$, alike, one may find $0 \leq \kappa \leq 1$. So (1) is preserved.

Now, for $\left(\max \left(\rho_{j}\right)\right)^{q}+\left(\max \left(\kappa_{j}\right)\right)^{q} \leq 1$, we have

$$
\begin{aligned}
& \cup_{\rho \in h}(\max (\rho))^{q}+\cup_{\kappa \in g}(\max (\kappa))^{q} \\
& =1-\left(1-\prod_{\substack{i, j=1 \\
i \neq j}}^{\tau}\left(1-\left(1-\max _{i}^{w_{i} q}\right)^{s}\left(1-\max _{j}^{w_{j} q}\right)^{t}\right)^{\frac{1}{\tau(\tau-1)}}\right)^{\frac{1}{s+t}} \\
& +\left(1-\prod_{\substack{i, j=1 \\
i \neq j}}^{\tau}\left(1-\left(1-\left(1-\max _{i}^{q}\right)^{w_{i}}\right)^{s}\left(1-\left(1-\max _{j}^{q}\right)^{w_{j}}\right)^{t}\right)^{\frac{1}{\tau(\tau-1)}}\right)^{\frac{1}{s+t}} \\
& \leq\left(1-\prod_{\substack{i, j=1 \\
i \neq j}}^{\tau}\left(1-\left(1-\left(1-\max _{i}^{q}\right)^{w_{i}}\right)^{s}\left(1-\left(1-\max _{j}^{q}\right)^{w_{j}}\right)^{t}\right)^{\left.\frac{1}{\tau(\tau-1)}\right)^{\frac{1}{s+t}}}\right. \\
& 1-\left(1-\prod_{\substack{\tau, j=1 \\
i \neq j}}^{\tau}\left(1-\left(1-\left(1-\max _{i}^{q}\right)^{w_{i}}\right)^{s}\left(1-\left(1-\max _{j}^{q}\right)^{w_{j}}\right)^{t}\right)^{\frac{1}{\tau(\tau-1)}}\right)^{\frac{1}{s+t}} \\
& =1
\end{aligned}
$$

So (1) is preserved.

By adjusting the estimations of parameter $s, t$ and $q$, some unique instances of q-RODHFWBM operator are discussed as pursues.

(1) For some particular values of $q$, there exist following vital cases. 
Remark 19. When $q=1$, the q-RODHFWDBM operator will turn into dual hesitant fuzzy weighted dual Bonferroni mean (DHFWDBM) operator as shown below:

$$
\begin{aligned}
& q-\operatorname{RODHFWDBM} M^{s, t}\left(d_{1}, d_{2}, \ldots, d_{\tau}\right)=\frac{1}{s+t}\left(\otimes_{i, j=1}^{\tau}{ }_{i \neq j}\left(s d_{i}^{w_{i}} \oplus t d_{j}^{w_{j}}\right)\right)^{\frac{1}{\tau(\tau-1)}} \\
& =\cup_{\rho_{i} \in h_{i}, \kappa_{i} \in g_{i}, \rho_{j} \in h_{j}, \kappa_{j} \in g_{j}}\left\{1-\left(1-\prod_{\substack{i, j=1 \\
i \neq j}}^{\tau}\left(1-\left(1-\rho_{i}^{w_{i} q}\right)^{s}\left(1-\rho_{j}^{w_{j} q}\right)^{t}\right)^{\frac{1}{\tau(\tau-1)}}\right)^{\frac{1}{s+t}},\right. \\
& \left.\left(1-\prod_{\substack{i, j=1 \\
i \neq j}}^{\tau}\left(1-\left(1-\left(1-\kappa_{i}^{q}\right)^{w_{i}}\right)^{s}\left(1-\left(1-\kappa_{j}^{q}\right)^{w_{j}}\right)^{t}\right)^{\frac{1}{\tau(\tau-1)}}\right)^{\frac{1}{s+t}}\right\}
\end{aligned}
$$

Remark 20. When $q=2$, the q-RODHDFWDBM operator will turn into dual hesitant Pythagorean fuzzy weighted DBM (DHPFWDBM), as defined

$$
\begin{aligned}
& \operatorname{DHFWDBM} M^{s, t}\left(d_{1}, d_{2}, \ldots, d_{\tau}\right)=\frac{1}{s+t}\left(\otimes_{\substack{i, j=1 \\
i \neq j}}^{\tau}\left(s d_{i}^{w_{i}} \oplus t d_{j}^{w_{j}}\right)\right)^{\frac{1}{\tau(\tau-1)}} \\
& =\cup_{\rho_{i} \in h_{i}, \kappa_{i} \in g_{i}, \rho_{j} \in h_{j}, \kappa_{j} \in g_{j}}\left\{\sqrt{1-\left(1-\prod_{\substack{i, j=1 \\
i \neq j}}^{\tau}\left(1-\left(1-\rho_{i}^{2 w_{i}}\right)^{s}\left(1-\rho_{j}^{2 w_{j}}\right)^{t}\right)^{\frac{1}{\tau(\tau-1)}} \frac{1}{s+t}\right.},\right. \\
& \left.\left(\sqrt{1-\prod_{\substack{i, j=1 \\
i \neq j}}^{\tau}\left(1-\left(1-\left(1-\kappa_{i}^{2}\right)^{w_{i}}\right)^{s}\left(1-\left(1-\kappa_{j}^{2}\right)^{w_{j}}\right)^{t}\right)^{\frac{1}{\tau(\tau-1)}}}\right)^{\frac{1}{s+t}}\right\}
\end{aligned}
$$

(2) For parameter $s$ and $t$, there exist the following vital cases.

Remark 21. When $t \rightarrow 0$, the q-RODHFWDBM will turn into the q-rung orthopair dual hesitant fuzzy weighted dual arithmetic mean (q-RODHFWDAM) operator as shown below:

$$
\begin{aligned}
& \frac{1}{s+t}\left(\otimes_{\substack{i, j=1 \\
i \neq j}}^{\tau}\left(s d_{i}^{w_{i}} \oplus t d_{j}^{w_{j}}\right) \frac{1}{\tau(\tau-1)}\right)=\cup_{\rho_{i} \in h_{i}, \kappa_{i} \in g_{i}, \rho_{j} \in h_{j}, \kappa_{j} \in g_{j}} \\
& \left\{\sqrt[q]{1-\left(1-\prod_{\substack{i, j=1 \\
i \neq j}}^{\tau}\left(1-\left(1-\rho_{i}^{w_{i} q}\right)^{s}\right) \frac{1}{\tau}\right) \frac{1}{s}},\left(\sqrt[q]{1-\prod_{\substack{i, j=1 \\
i \neq j}}^{\tau}\left(1-\left(1-\left(1-\kappa_{i}^{q}\right)^{w_{i}}\right)^{s}\right) \frac{1}{\tau}} \frac{1}{s}\right\}\right.
\end{aligned}
$$

Remark 22. When $s=1$ and $t \rightarrow 0$, the q-RODHFWDBM will turn into the q-rung orthopair dual hesitant fuzzy weighted dual geometric mean (q-RODHFWDGM) operator as shown below:

$$
\begin{aligned}
& \frac{1}{s+t}\left(\otimes_{\substack{i, j=1 \\
i \neq j}}^{\tau}\left(s d_{i}^{w_{i}} \oplus t d_{j}^{w_{j}}\right)^{\frac{1}{\tau(\tau-1)}}\right)=\cup_{\rho_{i} \in h_{i}, \kappa_{i} \in g_{i}, \rho_{j} \in h_{j}, \kappa_{j} \in g_{j}} \\
& \left\{\sqrt[q]{\prod_{\substack{i, j=1 \\
i \neq j}}^{\tau}\left(\rho_{i}^{w_{i} q}\right) \frac{1}{\tau}},\left(\sqrt[q]{1-\prod_{\substack{i, j=1 \\
i \neq j}}^{\tau}\left(\left(1-\kappa_{i}^{q}\right)^{w_{i}}\right) \frac{1}{\tau}} \frac{1}{s}\right\}\right.
\end{aligned}
$$


Remark 23. When $s=2$ and $t \rightarrow 0$, the q-RODHFWDBM will turn into the q-rung orthopair dual hesitant fuzzy weighted dual square mean (q-RODHFWDSM) operator as shown below:

$$
\begin{aligned}
& \frac{1}{s+t}\left(\otimes_{\substack{i, j=1 \\
i \neq j}}^{\tau}\left(s d_{i}^{w_{i}} \oplus t d_{j}^{w_{j}}\right)^{\frac{1}{\tau(\tau-1)}}\right)=\cup_{\rho_{i} \in h_{i}, \kappa_{i} \in g_{i}, \rho_{j} \in h_{j}, \kappa_{j} \in g_{j}}
\end{aligned}
$$

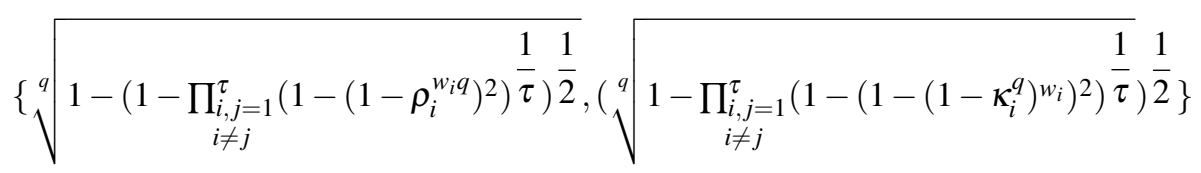

Remark 24. When $s=1$ and $t=1$, the q-RODHFWDBM will turn into the q-rung orthopair dual hesitant fuzzy weighted dual interrelated square mean (q-RODHFWDISM) operator as shown below:

$$
\begin{aligned}
& q-\operatorname{RODHFWDISM} M^{1,1}\left(d_{1}, d_{2}, \ldots, d_{\tau}\right)=\frac{1}{s+t}\left(\otimes_{\substack{i, j=1 \\
i \neq j}}^{\tau}\left(d_{i}^{w_{i}} \oplus d_{j}^{w_{j}}\right)\right) \frac{1}{\tau(\tau-1)} \\
& =\cup_{\rho_{i} \in h_{i}, \kappa_{i} \in g_{i}, \rho_{j} \in h_{j}, \kappa_{j} \in g_{j}}\left\{\sqrt[q]{1-\left(1-\prod_{\substack{i, j=1 \\
i \neq j}}^{\tau}\left(1-\left(1-\rho_{i}^{w_{i} q}\right)\left(1-\rho_{j}^{w_{j} q}\right)\right)^{\frac{1}{\tau(\tau-1)}}\right)^{\frac{1}{2}}},\right. \\
& \left(\sqrt[q]{\left.\left.1-\prod_{\substack{i, j=1 \\
i \neq j}}^{\tau}\left(\oint 1-\left(1-\left(1-\kappa_{i}^{q}\right)^{w_{i}}\right)\left(1-\left(1-\kappa_{j}^{q}\right)^{w_{j}}\right)\right)^{\frac{1}{\tau(\tau-1)}}\right)^{\frac{1}{2}}\right\}}\right.
\end{aligned}
$$

Example 2.4. Let $d_{1}=\{\{0.3,0.4\},\{0.5\}\}, d_{2}=\{\{0.7\},\{0.1,0.2,0.6\}\}$, and $d_{3}=\{\{0.6\},\{0.3\}\}$ be three q-RODHFNs, and let $s=1, t=1$ and $q=3$ then utilizing Eq.(35), we have for the membership (favorable) function $\rho$, the final values are as below.

Alike, we can find $\rho_{2}=q-\operatorname{RODHFDWBM}(0.4,0.7,0.6)=0.9999$, and $\rho=\{0.9287,0.9999\}$. For the non-membership (unfavorable) function $\kappa$, the ultimate values are shown below. Alike, the results of $\kappa_{2}$, and $\kappa_{3}$, are $\kappa_{2}=\mathrm{q}-\mathrm{RODHFDWBM}(0.5$, $0.2,0.3)=0.9980, \kappa_{3}=q-\operatorname{RODHFDWBM}(0.5,0.6,0.3)=0.9856$, so we can list $\kappa=\{0.9952,0.9980,0.9856\}$. Therefore,

$$
q-\operatorname{RODHFDWBM}\left(d_{1}, d_{2}, d_{3}\right)=\{\{0.9287,0.9999\},\{0.9952,0.9980,0.9856\}\}
$$

\section{MODELS FOR MADM WITH q-RODHFNs}

In the light of the q-RODHFWBM and q-RODHFWDBM operators, we shall furnish the model for MADM with qRODHFNs. Let $O=\left\{O_{1}, O_{2}, \ldots, O_{m}\right\}$ be a discrete set of alternatives, and $K=\left\{K_{1}, K_{2}, \ldots, K_{\tau}\right\}$ be collection of attributes, $w=\left\{w_{1}, w_{2}, \ldots, w_{\tau}\right\}$ is the weight vector of the attribute $K_{j}(j=1,2, \ldots, \tau)$ where $0 \leq w_{j} \leq 1, \sum_{j=1}^{\tau} w_{j}=1$. Suppose that $d=\left(d_{i j}\right)_{m \times \tau}=\left(h_{i j}, g_{i j}\right)_{m \times \tau}$ is the q-rung orthopair fuzzy decision matrix, where $h_{i j}$ set specify the level that the alternative $O_{i}$ satisfy the attribute $K_{j}$ given by the decision maker, $g_{i j}$ set specify the level that the alternative $O_{i}$ doesn't satisfy the attribute $K_{j}$ given by the decision maker, $\rho_{i j} \in h_{i j} \subset[0,1], \kappa_{i j} \in g_{i j} \subset[0,1],\left(\rho_{i j}\right)^{2}+\left(\kappa_{i j}\right)^{2} \leq 1, i=1,2, \ldots, m, j=1,2, \ldots, \tau$. In the accompanying, we will utilize the q-RODHFWBM and q-RODHFWDBM operator to the MADM problems for q-RODHFNs.

Step 1 : We take advantage of q-RODHFNs of the matrix $\widetilde{U}$, and utilize q-RODHFWBM operator to acquire $d_{i}(i=$ $1,2, \ldots, m)$ of the alternative $O_{i}$.

Step 2 : Determine the scores $L\left(d_{i}\right)(i=1,2, \ldots, m)$ of the whole collection of q-RODHFNs $d_{i}(i=1,2, \ldots, m)$ and finally rank all the alternatives $O_{i}(i=1,2, \ldots, m)$ and then choose the exclusively optimal one(s). If the score values of two $L\left(d_{i}\right)$ and $L\left(d_{k}\right)$ are same, then we shall utilize the accuracy values $T\left(d_{i}\right)$ and $T\left(d_{k}\right)$ of the whole collection of q-RODHFNs and $d_{i}$ and $d_{k}$, respectively, and then arrange the alternatives $O_{i}$ and $O_{k}$ with respect to the accuracy degrees $T\left(d_{i}\right)$ and $T\left(d_{k}\right)$

Step 3 : Arrange all the alternatives $O_{i}(i=1,2, \ldots, m)$

in descending order and select the optimal one(s) likewise $L\left(d_{i}\right)(i=1,2, \ldots, m)$

Step 4 : End. 


\section{Application and Comparative Analysis}

\subsection{Numerical Example}

In this segment, we shall furnish an application to choose green providers in green inventory network the board (GINB) with q-RODHFNs. There are five possible green providers in GINB $O_{i}(i=1,2,3,4,5)$ to decide. The specialists evaluate the five potential green providers with respect to the following attributes: $1 . K_{1}$ is the item quality factor; $2 . K_{2}$ is natural factors; $3 . K_{3}$ is conveyance factor; $4 . K_{4}$ is value factor. Five green providers $O_{i}(i=1,2,3,4,5)$ are to be classified under q-RODHFNs with respect to four attributes with weight vector $w=(0.4,0.3,0.1,0.2)$ displayed in Table 1.

Table 1. q-RODHFN decision matrixi $(\widetilde{U})$

\begin{tabular}{cllll}
\hline & $K_{1}$ & $K_{2}$ & $K_{3}$ & $K_{4}$ \\
\hline$O_{1}$ & $\{(0.5,0.6\},(0.4\}\}$ & $\{\{0.2,0.3\},\{0.4,0.6\}\}$ & $\{(0.1,0.4\},(0.3\}\}$ & $\{(0.2,0.4\},(0.6\}\}$ \\
$O_{2}$ & $\{\{0.7\},\{0.2\}\}$ & $\{\{0.5,0.6,0.8\},\{0.2\}\}$ & $\{(0.7\},(0.3,0.4,0.5\}\}$ & $\{\{0.4\},\{0.2,0.3\}\}$ \\
$O_{3}$ & $\{\{0.6,0.8\},\{0.5\}\}$ & $\{\{0.5\},\{0.1,0.4\}$ & $\{\{0.1,0.4,0.5\},\{0.2\}\}$ & $\{\{0.3,0.4,0.5\},\{0.4\}\}$ \\
$O_{4}$ & $\{\{0.2\},\{0.4\}\}$ & $\{\{0.4,0.5,0.6\},\{0.7\}\}$ & $\{\{0.2,0.4\},\{0.5\}\}$ & $\{\{0.2\},\{0.3,0.6,0.7\}\}$ \\
$O_{5}$ & $\{\{0.4,0.5\},\{0.4\}\}$ & $\{\{0.5,0.6,0.7\},\{0.6\}\}$ & $\{\{0.2,0.3\},\{0.5\}$ & $\{\{0.1,0.4,0.5\},\{0.2\}\}$ \\
\hline
\end{tabular}

In the accompanying, we take the advantage of the operators developed for provider selection in provide network board with q-rung orthopair dual hesitant fuzzy numbers (q-RODHFNs) datum.

Step 1: We take advantage of the decision datum in matrix $\widetilde{U}$, and the q-RODHFWBM operator to collect the collective preference values $d_{i}$ of the provider in green inventory network the board $O_{i}(i=1,2,3,4,5)$. The collective preference values $d_{i}$ of the provider in green inventory network the board $O_{i}(i=1,2,3,4,5)$ are listed below

$$
\begin{aligned}
& d_{1}=q-\text { RODHFWBM } M_{w}^{1,1}\left(d_{11}, d_{12}, d_{13}, d_{14}\right)=\left(\frac{1}{12}\left\{\oplus_{i, j=1}^{4}\left(w_{i} d_{i}\right)^{1} \otimes\left(w_{j} d_{j}\right)^{1}\right\}\right)^{\frac{1}{2}} \\
&= \cup_{\rho_{i} \in h_{i}, \rho_{j} \in h_{j}, \kappa_{i} \in g_{i}, \kappa_{j} \in g_{j}}\left\{\left(\sqrt[3]{1-\prod_{\substack{i, j=1 \\
i \neq j}}^{4}\left(1-\left(1-\left(1-\rho_{i}^{3}\right)^{w_{i}}\right)\left(1-\left(1-\rho_{j}^{3}\right)^{w_{j}}\right)\right)^{\frac{1}{24}}}\right)^{\frac{1}{12}}\right. \\
&\left.\sqrt[3]{1-\left(1-\prod_{i, j=1}^{4}\left(1-\left(1-\kappa_{i}^{3 w_{i}}\right)\left(1-\kappa_{j}^{3 w_{j}}\right)\right)^{\frac{1}{24}}\right)^{\frac{1}{2}}}\right\} \\
&=\{\langle\{0.5,0.6\},\{0.4\}\rangle,\langle\{0.2,0.3\},\{0.4,0.6\}\rangle,\langle\{0.1,0.4\},\{0.3\}\rangle,\langle\{0.2,0.4\},\{0.6\}\rangle\} \\
&=\{\{0.1620,0.2031,0.1906,0.2176,0.1876,0.2159,0.2060,0.2279, \\
&0.1782,0.2231,0.2091,0.2378,0.2062,0.2361,0.2255,0.2483\},\{0.8782,0.8948\}\} \\
&=\{\langle\{0.7\},\{0.2\}\rangle,\langle\{0.5,0.6,0.8\},\{0.2\}\rangle,\langle\{0.7\},\{0.3,0.4,0.5\}\rangle,\langle\{0.4\},\{0.2,0.3\}\rangle\} \\
&=\{\{0.3390,0.3589,0.4077\},\{0.8011,0.8122,0.8135,0.8246,0.8073,0.8196\}\} \\
& d_{3}= q-R O D H F W B M_{w}^{1,1}\left(d_{31}, d_{32}, d_{33}, d_{34}\right) \\
&=\{\langle\{0.6,0.8\},\{0.5\}\rangle,\langle\{0.5\},\{0.1,0.4\}\rangle,\langle\{0.1,0.4,0.5\},\{0.2\}\rangle,\langle\{0.3,0.4,0.5\},\{0.4\}\rangle\} \\
&=\{\{0.2671,0.2766,0.2899,0.2754,0.2841,0.3366, \\
& 0.3216,0.3135,0.3302,0.3427,0.3487,0.3486, \\
&0.2964,0.2830,0.2909,0.3025,0.3230,0.3370\},\{0.8373,0.8653\}\}
\end{aligned}
$$




$$
\begin{aligned}
& d_{4}=q-\text { RODHFWBM } M_{w}^{1,1}\left(d_{41}, d_{42}, d_{43}, d_{44}\right) \\
& =\{\langle\{0.2\},\{0.4\}\rangle,\langle\{0.4,0.5,0.6\},\{0.7\}\rangle,\langle\{0.2,0.4\},\{0.5\}\rangle,\langle\{0.2\},\{0.3,0.6,0.7\}\rangle\} \\
& =\{\{0.1849,0.1465,0.1667,0.1634,0.1796,0.2974\},\{0.8373,0.8653\}\} \\
& d_{5}=q-\text { RODHFWBM }{ }_{w}^{1,1}\left(d_{51}, d_{52}, d_{53}, d_{54}\right) \\
& =\{\langle\{0.4,0.5\},\{0.4\}\rangle,\langle\{0.5,0.6,0.7\},\{0.6\}\rangle,\langle\{0.2,0.3\},\{0.5\}\rangle,\langle\{0.1,0.4,0.5\},\{0.2\}\rangle\} \\
& =\{\{0.2134,0.2721,0.2997,0.3007,0.2705,0.2683, \\
& 2535,02333,02542,02743,02552,02892, \\
& 0.2914,0.2769,0.2710,0.2576,0.2609,0.2843, \\
& 0.2988,0.3107,0.3125,0.3008,0.2867,0.2734, \\
& 0.2171,0.2368,0.2562,0.2370,0.2402,0.2891, \\
& 0.2688,0.2635,0.2766,0.2577,0.2788,0.2910\},\{0.8741\}\}
\end{aligned}
$$

Step 2: Find the scores $S\left(O_{i}\right)(i=1,2,3,4,5)$ of the collective q-rung orthopair dual hesitant fuzzy values $O_{i}(i=1,2,3,4,5)$ :

$$
\begin{aligned}
& S\left(O_{1}\right)=0.1564, S\left(O_{2}\right)=0.2566, S\left(O_{3}\right)=0.2064 \\
& S\left(O_{4}\right)=0.1953, S\left(O_{5}\right)=0.1759
\end{aligned}
$$

Step 3: Rank all the providers $O_{i}(i=1,2,3,4,5)$ likewise the scores $S\left(O_{i}\right)(i=1,2,3,4,5)$ of the collective q-rung orthopair dual hesitant fuzzy numbers: $\mathrm{O}_{2} \succ \mathrm{O}_{3} \succ \mathrm{O}_{4} \succ \mathrm{O}_{5} \succ \mathrm{O}_{1}$, and thus the most desirable supplier is $\mathrm{O}_{2}$.

Based on the q-RODHFWDBM operator, in order to select the most desirable supplier, we can develop an approach to multiple attribute decision making problems with q-rung orthopair dual hesitant fuzzy information, which can be described as following:

Step 1 : Aggregate all q-rung orthopair dual hesitant fuzzy value $d_{i j}(j=1,2,3,4)$ by using the dual hesitant q-rung orthopair fuzzy weighted DBM (q-RODHFWDBM) operator to derive the overall q-rung orthopair dual hesitant fuzzy values $d_{i}(i=1,2,3,4,5)$ of the supplier $A_{i}$. The overall performance values of all the supplier $A_{1}$ (here, we take $\left.\mathrm{q}=3, \mathrm{~s}=1, \mathrm{t}=1\right)$ are given below,

Step 2 : Calculate the scores $s\left(A_{i}\right)(i=1,2,3,4,5)$ of the overall q-rung orthopair dual hesitant fuzzy values $A_{i}(i=1,2,3,4,5)$ of the supplier $A_{i}$ :

$$
S\left(A_{1}\right)=0.4297, S\left(A_{2}\right)=0.5378, S\left(A_{3}\right)=0.5021 S\left(A_{4}\right)=0.3839, S\left(A_{5}\right)=0.3839
$$

Step $\widetilde{3}$ : Rank all the suppliers in supply chain management $A_{i}(i=1,2,3,4,5)$ in accordance with the scores $s\left(A_{i}\right)(i=$ $1,2,3,4,5)$ of the overall dual hesitant q-rung orthopair fuzzy values $A_{i}(i=1,2,3,4,5)$ by using definition 2.15 : $A_{2} \succ A_{3} \succ$ $A_{5} \succ A_{1} \succ A_{4}$, and thus the most desirable supplier is $A_{2}$. From the above analysis, it is easily seen that although the overall rating values of the alternatives are same by using two operators respectively.

\subsection{Comparative analysis compared with existing magdm methods}

To demonstrate the superiorities of the proposed method, we have compared our method with that (1) developed by Wang et al.s ${ }^{(26)}$ based on the dual hesitant fuzzy weighted averaging (DHFWA) operator, (2) presented by Tu et al.s ${ }^{(27)}$, based on the dual hesitant fuzzy weighted Bonferroni mean (DHFWBM) operator, (3) putforwarded by Tang ${ }^{(24)}$, based on the dual hesitant Pythagorean fuzzy Heronian weighted averaging (DHPFHWA) operator, (4) proposed by, Xu et al.s ${ }^{(23)}$ based on the 
Table 2. Score functions and ranking results..

\begin{tabular}{|c|c|c|}
\hline Methods & Score Function $\mathrm{s}\left(\mathrm{d}_{i}\right)(\mathrm{i}=1,2,3,4,5)$ & Ranking Results \\
\hline $\begin{array}{l}\text { Wang et al.'(26) method based on the } \\
\text { DHFWA operator }\end{array}$ & $\begin{array}{l}\mathrm{s}\left(\mathrm{A}_{1}\right)=0.3195 \\
\mathrm{~s}\left(\mathrm{~A}_{2}\right)=0.4117 \\
\mathrm{~s}\left(\mathrm{~A}_{3}\right)=0.3753 \\
\mathrm{~s}\left(\mathrm{~A}_{4}\right)=0.1918 \\
\mathrm{~s}\left(\mathrm{~A}_{5}\right)=0.1189\end{array}$ & $A_{2} \succ A_{1} \succ A_{5} \succ A_{3} \succ A_{4}$ \\
\hline $\begin{array}{l}\text { Tu et al.s }{ }^{(27)} \text { method based on the } \\
\text { DHFWBM operator }\end{array}$ & $\begin{array}{l}\mathrm{s}\left(\mathrm{A}_{1}\right)=-0.3821 \\
\mathrm{~s}\left(\mathrm{~A}_{2}\right)=-0.3186 \\
\mathrm{~s}\left(\mathrm{~A}_{3}\right)=-0.3850 \\
\mathrm{~s}\left(\mathrm{~A}_{4}\right)=-0.4743 \\
\mathrm{~s}\left(\mathrm{~A}_{5}\right)=0.1189\end{array}$ & $A_{2}>A_{3}>A_{4}>A_{1}>A_{5}$ \\
\hline $\begin{array}{l}\text { Tang et al's }{ }^{(24)} \text { method based on the } \\
\text { DHPFHWA operator }\end{array}$ & $\begin{array}{l}\mathrm{s}\left(\mathrm{A}_{1}\right)=0.3142 \\
\mathrm{~s}\left(\mathrm{~A}_{2}\right)=0.3014 \\
\mathrm{~s}\left(\mathrm{~A}_{3}\right)=0.2968 \\
\mathrm{~s}\left(\mathrm{~A}_{4}\right)=0.0268 \\
\mathrm{~s}\left(\mathrm{~A}_{5}\right)=0.1197\end{array}$ & $A_{2} \succ A_{3} \succ A_{5} \succ A_{1} \succ A_{4}$ \\
\hline $\begin{array}{l}\mathrm{Xu} \text { et al.' } \mathrm{s}^{(23)} \text { method based on theq- } \\
\text { RDHFWHM operator }(\mathrm{s}=\mathrm{t}=2)\end{array}$ & $\begin{array}{l}\mathrm{s}\left(\mathrm{A}_{1}\right)=0.2359 \\
\mathrm{~s}\left(\mathrm{~A}_{2}\right)=0.2187 \\
\mathrm{~s}\left(\mathrm{~A}_{3}\right)=0.1284 \\
\mathrm{~s}\left(\mathrm{~A}_{4}\right)=0.0034 \\
\mathrm{~s}\left(\mathrm{~A}_{5}\right)=0.1198\end{array}$ & $A_{2} \succ A_{3} \succ A_{5} \succ A_{1} \succ A_{4}$ \\
\hline The proposed method in this paper & $\begin{array}{l}s\left(A_{1}\right)=0.4297 \\
s\left(A_{2}\right)=0.5378 \\
s\left(A_{3}\right)=0.5021 \\
s\left(A_{4}\right)=0.3839 \\
s\left(A_{5}\right)=0.3839\end{array}$ & $A_{2} \succ A_{3} \succ A_{5} \succ A_{1} \succ A_{4}$ \\
\hline
\end{tabular}

dual hesitant Pythagorean fuzzy Heronian weighted averaging (DHPFHWA) operator. We utilized these methods to solve the above example, and the score functions and ranking results can be found in Table 2.

First of all, Wang et al.s ${ }^{(26)}$ and Tu et al.s. ${ }^{(27)}$ methods are based on DHFSs. Tang et al.'s ${ }^{(24)}$ method is based on DHPFSs. As mentioned above, DHFS and DHPFS are two special cases of $\mathrm{q}$-RDHFS. When $\mathrm{q}=1$, then $\mathrm{q}$-RDHFS is reduced to DHFS, and when $\mathrm{q}=2$, $\mathrm{q}$-RDHFS is reduced to DHPFS. Evidently, q-RDHFS is more general and can describe a greater information range and process more information in the process of MAGDM. For instance, if an attribute value provided by DMs is $\{\{0.1$, $0.2,0.6,0.7\},\{0.1,0.4,0.5\}\}$, then obviously, the pair $\{\{0.1,0.2,0.6,0.7\},\{0.1,0.4,0.5\}\}$ is not valid for DHFSs and DHPFSs. Thus, our method is more general, powerful, and can process more information in MAGDM. Wang et al.s ${ }^{(26)}$ method is based on the simple weighted averaging operator. The drawback of this methods is that it does not consider the interrelationship between arguments. In other words, they assume all attributes are independent, which is not correct to some extent. In the abovementioned example, when choosing the most appropriate supplier, we need to consider not only the attribute values of each supplier but also the correlation between these attributes. Thus, Wang et al's ${ }^{(26)}$ method is not suitable for dealing with this problem. As our method has the ability to capture variable correlations, it is more reasonable than Wang et al's method for addressing this problem. Xu et al.s ${ }^{(23)}$ is based on HM. Tu et al.'s ${ }^{(27)}$ and our methods based on Bonferroni mean (BM). The prominent characteristic of BM and HM is that both can consider the interrelationship between arguments. Therefore, all the three can process the interrelationship among attribute values. However, Xu et al.s ${ }^{(23)}$ method and ours are better than Tu et al's ${ }^{(27)}$ method. In addition, as Tu et al.s ${ }^{(27)}$ is a special case of our method (when $\mathrm{q}=1$ ), our method is more general, scientific, and applicable than Tu et al.s ${ }^{(27)}$ method.

\section{Conclusion}

In this article, we have examined the MADM problems under q-RODHFNs. we have utilized the BM operator and established some BM aggregation operators with q-RODHFNs. We have developed (q-RODHFBM) operator, (q-RODHFWBM) operator, DBM operator, (q-RODHFDBM) operator and (q-RODHFWDBM) operator. Also, the important merits of the examined operators are talked about. Furthermore, we have endorsed q-RODHFWBM and q-RODHFWDBM operators to construct decision-making steps to handle the q-rung orthopair dual hesitant fuzzy MADM problems. Finally, we take a solid example 
for examining the green provider selection to exhibit our established model and to assert its efficiency and objectiveness. We have compared our results with q-RODHFWHM and q-RODHFWGHM operators, despite the fact that the results are minimal extraordinary and the ideal option is not changed. However, the q-RODHFWHM and q-RODHFWGHM operators just include the interrelationship of two arbitrary numbers but our introduced operators can include the interrelationship of any number arbitrary arguments, that indicates our established method is more decisive to handle the MADM problems. In the forthcoming, we will maintain our study about the MADM issues with the application and expansion of the presented operators to other realm.

\section{References}

1) Atanassov KT. Intuitionistic fuzzy sets. In: Intuitionistic fuzzy sets 1999. Heidelberg. Physica. ;p. 1-137.

2) Zadeh LA. Fuzzy sets. Information and Control. 1965;8:338-353. Available from: https://dx.doi.org/10.1016/s0019-9958(65)90241-x.

3) Torra V. Hesitant fuzzy sets. International Journal of Intelligent Systems. 2010;25(6). Available from: https://dx.doi.org/10.1002/int.20418.

4) Peng X, Yang Y. Some results for Pythagorean fuzzy sets. International Journal of Intelligent Systems. 2015;30(11):991-1029.

5) Wei GW. Pythagorean fuzzy Hamacher power aggregation operators in multiple attribute decision making. Fundamenta Informaticae. 2019;166(1):57-85.

6) Peng X, Yuan H, Yang Y. Pythagorean fuzzy information measures and their applications. International Journal of Intelligent Systems. 2017;32(10):9911029.

7) Wei G. Some cosine similarity measures for picture fuzzy sets and their applications to strategic decision making. Informatica. 2017;28(3):547-564.

8) Bonferroni C. Sulle medie multiple di potenze. Bollettino dell'Unione Matematica Italiana. 1950;5(3-4):267-270.

9) Liang D, Darko AP, Xu Z. Pythagorean Fuzzy Partitioned Geometric Bonferroni Mean and Its Application to Multi-criteria Group Decision Making with Grey Relational Analysis. International Journal of Fuzzy Systems. 2019;21(1):115-128. Available from: https://dx.doi.org/10.1007/s40815-018-0544-x.

10) Yager RR. Generalized Orthopair Fuzzy Sets. IEEE Transactions on Fuzzy Systems. 2017;25(5):1222-1230. Available from: https://dx.doi.org/10.1109/ tfuzz.2016.2604005.

11) Liu P, Wang P. Some q-rung orthopair fuzzy aggregation operators and their applications to multiple-attribute decision making. International Journal of Intelligent Systems. 2018;33(2):259-280.

12) Liu P, Chen SM, Wang P. Multiple-attribute group decision-making based on q-rung orthopair fuzzy power maclaurin symmetric mean operators. IEEE Transactions on Systems, Man, and Cybernetics: Systems. 2018.

13) Liu P, Wang P. Multiple-attribute decision-making based on Archimedean Bonferroni Operators of q-rung orthopair fuzzy numbers. IEEE Transactions on Fuzzy systems. 2018;27(5):834-848.

14) Wang P, Wang J, Wei G, Wei C. Similarity Measures of q-Rung Orthopair Fuzzy Sets Based on Cosine Function and Their Applications. Mathematics. 2019;7(4). Available from: https://dx.doi.org/10.3390/math7040340.

15) Peng X, Dai J, Garg H. Exponential operation and aggregation operator for q-rung orthopair fuzzy set and their decision-making method with a new score function. International Journal of Intelligent Systems. 2018;33(11):2255-2282. Available from: https://dx.doi.org/10.1002/int.22028.

16) Wei G, Gao H, Wei Y. Some q-rung orthopair fuzzy Heronian mean operators in multiple attribute decision making. International Journal of Intelligent Systems. 2018;33(7):1426-1458.

17) Wang J, Wei G, Lu J, Alsaadi FE, Hayat T, Wei C, et al. Some q-rung orthopair fuzzy Hamy mean operators in multiple attribute decision-making and their application to enterprise resource planning systems selection. International Journal of Intelligent Systems. 2019;34(10):2429-2458.

18) Liu P, Liu P, Wang P, Zhu B. An Extended Multiple Attribute Group Decision Making Method Based on q-Rung Orthopair Fuzzy Numbers. IEEE Access. 2019;7. Available from: https://dx.doi.org/10.1109/access.2019.2951357.

19) Liu P, Wang Y. Multiple attribute decision making based on q-rung orthopair fuzzy generalized Maclaurin symmetic mean operators. Information Sciences. 2020;518:181-210. Available from: https://dx.doi.org/10.1016/j.ins.2020.01.013.

20) Liu P, Ali Z, Mahmood T. A Method to Multi-Attribute Group Decision-Making Problem with Complex q-Rung Orthopair Linguistic Information Based on Heronian Mean Operators. International Journal of Computational Intelligence Systems. 2019;2019:1465-1496. Available from: https://dx.doi.org/10. 2991/ijcis.d.191030.002.

21) Liu P, Liu W. Multiple-attribute group decision-making method of linguisticq-rung orthopair fuzzy power Muirhead mean operators based on entropy weight. International Journal of Intelligent Systems. 2019;34(8):1755-1794. Available from: https://dx.doi.org/10.1002/int.22114.

22) Liu P, Liu W. Multiple-attribute group decision-making based on power Bonferroni operators of linguistic q-rung orthopair fuzzy numbers. International Journal of Intelligent Systems. 2019;34(4):652-689.

23) $\mathrm{Xu} \mathrm{Y}$, Shang $\mathrm{X}$, Wang J, Wu W, Huang $\mathrm{H}$. Some q-rung dual hesitant fuzzy Heronian mean operators with their application to multiple attribute group decision-making. Symmetry. 2018;10(10):472.

24) Tang M, Wang J, Lu J, Wei G, Wei C, Wei Y. Dual Hesitant Pythagorean Fuzzy Heronian Mean Operators in Multiple Attribute Decision Making. Mathematics. 2019;7(4):344-344. Available from: https://dx.doi.org/10.3390/math7040344.

25) Zhu B, Xu Z, Xia M. Dual Hesitant Fuzzy Sets. Journal of Applied Mathematics. 2012;2012:1-13. Available from: https://dx.doi.org/10.1155/2012/879629.

26) Wang H, Zhao X, Wei G. Dual hesitant fuzzy aggregation operators in multiple attribute decision making. Journal of Intelligent \& Fuzzy Systems. 2014;26(5):2281-2290.

27) Tu NH, Wang CY, Zhou XQ, Tao SD. Dual hesitant fuzzy aggregation operators based on Bonferroni means and their applications to multiple attribute decision making. Annl Fuzzy Math Inform. 2017;14:265-278.

28) Liu JB, Malik MA, Ayub N, Siddiqui HMA. Distance Measures for Multiple-Attributes Decision-Making Based on Connection Numbers of Set Pair Analysis With Dual Hesitant Fuzzy Sets. IEEE Access. 2020;8:9172-9184. Available from: https://dx.doi.org/10.1109/access.2019.2963484.

29) Wang J, Wang G, Wei C, Wei Y. Similarity Measures of q-Rung Orthopair Fuzzy Sets Based on Cosine Function and Their Applications. Mathematics. 2019;7. Available from: https://dx.doi.org/10.3390/math7040340. 